\title{
Archivos de Cardiología de México

\section{Reunión multidisciplinaria sobre el uso de los anticoagulantes orales directos en la fibrilación auricular no valvular}

\author{
Humberto Rodríguez-Reyes ${ }^{a, *}$, Antonio Arauz-Góngora ${ }^{\mathrm{b}}$, \\ Enrique Asensio-Lafuente ${ }^{c}$, Manuel de Jesús Celaya-Cota ${ }^{d}$, \\ Alejandro Cordero-Cabra ${ }^{e}$, Milton Guevara-Valdivia ${ }^{f}$, Raúl Izaguirre-Avila ${ }^{\mathrm{g}}$, \\ Susano Lara-Vaca ${ }^{\mathrm{h}}$, Vitelio Mariona-Moreno ${ }^{i}$, Enrique Martínez-Flores ${ }^{j}$, \\ Santiago Nava-Townsend ${ }^{k}$, Gerardo Pozas-Garza' y Gerardo Rodríguez-Diez ${ }^{m}$
}

\footnotetext{
a Sociedad Cardiovascular y Arritmias, Aguascalientes, México

b Clínica de Enfermedad Vascular Cerebral, Instituto Nacional de Neurología y Neurocirugía, Ciudad de México, México

' Departamento de Medicina Interna, Hospital Médica TEC 100, Querétaro, México

¿ Instituto de Corazón CIMA Hermosillo, Sonora, México

e Instituto Cardiovascular de Guadalajara, Guadalajara, México

f UMAE Hospital de Especialidades «Dr. Antonio Fraga Mouret», Centro Médico Nacional La Raza, Ciudad de México, México

" Departamento de Hematología, Clínica de anticoagulantes, Instituto Nacional de Cardiología «lgnacio Chávez»,

Ciudad de México, México

h Servicio de Arritmias, Centro Médico IMSS, León Guanajuato, México

i Servicio de Cardiología, Centro Médico Nacional de Occidente IMSS, Guadalajara, México

¡ Servicio de Electrofisiología, Centro Médico Nacional Siglo XXI, Ciudad de México, México

k Departamento de Electrocardiología, Instituto Nacional de Cardiología «lgnacio Chávez», Ciudad de México, México

' Instituto de Cardiología y Medicina vascular del TEC de Monterrey, Monterrey, México

m Departamento de Electrofisiología, Centro Médico Nacional 20 de Noviembre, Ciudad de México, México
}

Recibido el 22 de enero de 2016; aceptado el 21 de junio de 2016

\author{
PALABRAS CLAVE \\ Anticoagulantes \\ orales; \\ Fibrilación auricular;
}

\begin{abstract}
Resumen Conociendo el impacto real de la fibrilación auricular en el evento vascular cerebral, la Sociedad Mexicana de Electrofisiología y Estimulación Cardiaca (SOMEEC) contempló la iniciativa de desarrollar una reunión multidisciplinaria de expertos con la finalidad de actualizar la evidencia científica disponible a partir de guías de práctica clínica, metaanálisis y ensayos clínicos controlados, y complementarla con la experiencia y los puntos de vista de un grupo de expertos. Para cumplir con este objetivo, se reunió a un grupo de especialistas en el área de cardiología, electrofisiología, neurología y hematología que, dada su experiencia en ciertas áreas,
\end{abstract}

\footnotetext{
* Autor para correspondencia: Priv. Ipacaray n. ${ }^{\circ} 111-115$, Fracc. Las Américas, CP. 20230, Aguascalientes, México. Teléfono: +449 9162242; fax: +449 9154000; extensión 0.

Correo electrónico: humbertorodriguezr2@gmail.com (H. Rodríguez-Reyes).
} 
Sociedad Mexicana de Electrofisiología y Estimulación Cardiaca; Apixabán; Dabigatrán; Rivaroxabán; México

\section{KEYWORDS}

Oral anticoagulants; Atrial fibrillation; Sociedad Mexicana de Electrofisiología y Estimulación Cardiaca; Apixaban; Dabigatran; Rivaroxaban; Mexico compartieron la evidencia científica disponible ante el panel de expertos para dejar abierta una discusión sobre la información que se presentaría en el presente artículo. Este documento reúne la mejor evidencia científica disponible y pretende ser una herramienta útil que agilice la toma de decisiones para uso de los nuevos anticoagulantes orales en fibrilación auricular no valvular y cardiopatía isquémica, o referente al manejo de pacientes que presentan evento vascular cerebral, o insuficiencia renal, e incluso en aquellos que serán sometidos a procedimientos invasivos y cirugía electiva. En la misma se manejan esquemas comparativos de seguimiento y tratamiento que simplifica la toma de decisión por los especialistas participantes.

(c) 2016 Instituto Nacional de Cardiología Ignacio Chávez. Publicado por Masson Doyma México S.A. Este es un artículo Open Access bajo la licencia CC BY-NC-ND (http://creativecommons. org/licenses/by-nc-nd/4.0/).

Multidisciplinary meeting about the use of direct oral anticoagulants in nonvalvular atrial fibrillation

\begin{abstract}
Knowing the real impact of atrial fibrillation in the stroke, the Sociedad Mexicana of Electrofisiología y Estimulación Cardiaca (SOMEEC) had the initiative to develop a multidisciplinary meeting of experts the with the purpose to update the available scientific evidence from clinical practice guidelines, meta-analyses, controlled clinical trials, and complementing with the experience and views of a group of experts. To meet this goal, SOMEEC gathered a group of specialists in the area of cardiology, electrophysiology, neurology and hematology that given their experience in certain areas, they share the scientific evidence with the panel of experts to leave open a discussion about the information presented in this article. This document brings together the best scientific evidence available and aims to be a useful tool in the decision to use of new oral anticoagulants in nonvalvular atrial fibrillation and ischemic heart disease, or relating to the management of patients with stroke or renal failure, and even those that will be submitted to elective surgery and invasive procedures. In the same, they handled comparative schemes of follow-up and treatment which simplifies the decision making by the specialists participants.

(c) 2016 Instituto Nacional de Cardiología Ignacio Chávez. Published by Masson Doyma México S.A. This is an open access article under the CC BY-NC-ND license (http://creativecommons. org/licenses/by-nc-nd/4.0/).
\end{abstract}

\section{Introducción}

La fibrilación auricular (FA) es el trastorno del ritmo cardiaco más común, con una prevalencia del 1 al $2 \%$. Su incidencia aumenta con la edad, ya que más de una tercera parte son mayores de 80 años, aproximadamente el $12 \%$ tienen de 75 a 84 años y alrededor del $1 \%$ son $<60$ años ${ }^{1,2}$. En el primer registro mexicano de FA (ReMeFa), que incluyó 1,193 pacientes recientemente diagnosticados con FA, la edad promedio reportada fue de $65.9 \pm 13.4 \mathrm{annos}^{3}$.

La FA no valvular (FANV) es un factor de riesgo importante para presentar evento vascular cerebral (EVC) isquémico y embolismo sistémico (ES), provocando un aumento en la morbimortalidad ${ }^{4}$.

El EVC es la complicación más importante, con un riesgo relativo (RR) de 2 a 7 veces mayor que el de los pacientes sin FA. Además, los EVC asociados a FANV son especialmente severos, tienen mayor recurrencia y morbimortalidad que los EVC asociados a enfermedad aterotrombótica, generando un alto costo por el tratamiento individualizado y sus complicaciones ${ }^{4,5}$.

Se ha demostrado que el tratamiento con anticoagulantes orales directos (AOD) reduce la mortalidad por todas las causas en un 10\% (RR: 0.90; IC 95\%: 0.85-0.95; $\mathrm{p}=0.003)$ vs. warfarina en pacientes con $\mathrm{FA}^{6}$. Sin embargo, un gran porcentaje de pacientes reciben tratamiento subóptimo. Existen 2 escalas exclusivas para la estadificación de riesgo tromboembólico para pacientes con FA: $\mathrm{CHADS}_{2}$ y $\mathrm{CHA}_{2} \mathrm{DS}_{2}$-Vasc (Anexo 1,2), siendo esta última la recomendada actualmente por las guías, ya que es una versión perfeccionada de la $\mathrm{CHADS}_{2}$ que integra otros factores de riesgo de EVC. En base a la escala $\mathrm{CHA}_{2} \mathrm{DS}_{2}$-Vasc, los pacientes con puntuación $\geq 2$ deben recibir un anticoagulante oral $(\mathrm{AO})^{2}$. Los antagonistas de la vitamina $\mathrm{K}(\mathrm{AVK})$, como la warfarina, los inhibidores directos de la trombina (dabigatrán) y los inhibidores del factor $X$ activado (apixabán y rivaroxabán), son los AOD aprobados por la FDA para la prevención de eventos cardioembólicos en pacientes con FANV ${ }^{7}$.

\section{Tratamiento anticoagulante oral con antagonistas de la vitamina $\mathrm{K}$}

\section{Limitaciones y oportunidades perdidas}

Los AVK han sido usados durante más de 60 años y tienen numerosas ventajas, entre las que se incluyen múltiples estudios clínicos bien controlados que han dado evidencia 
de su beneficio ${ }^{8}$. Se ha probado la alta eficacia de la warfarina y de otros AVK (como acenocumarina) en la prevención de eventos tromboembólicos en pacientes con FANV paroxística, persistente o permanente, así como la reducción del riesgo de EVC en dos terceras partes de los pacientes aproximadamente ${ }^{4,9}$.

El tratamiento con AVK de alta calidad tiene como propósito obtener la máxima eficacia para prevenir eventos tromboembólicos, manteniendo un elevado índice de seguridad para prevenir las complicaciones hemorrágicas. Dentro de las ventajas se encuentra que su acción anticoagulante permanece por varios días y se puede graduar la intensidad de la anticoagulación, logrando obtener rápidamente un índice internacional normalizado (INR) en nivel terapéutico de 2 a $3^{5}$.

Para su prescripción se requiere experiencia y conocimiento por parte de los médicos involucrados, un sistema de evaluación organizado, la vigilancia del INR por medio de un laboratorio confiable, una buena comunicación con el paciente y un programa de educación continua tanto para los pacientes como para los diferentes profesionales que participan en la organización ${ }^{5}$. Este proceso incluye iniciar el tratamiento con dosis apropiadas para evitar las complicaciones hemorrágicas por la sobreanticoagulación en la primera semana de tratamiento. También se debe hacer una transición adecuada de los AVK con algún tipo de heparina que se haya empleado en el inicio del tratamiento (disminuyendo la actividad de los factores Ila, VIla, IXa y Xa), como ocurre en pacientes con trombosis venosa profunda o con embolia pulmonar.

Los AVK son medicamentos de bajo costo que no tienen efecto de reacción inversa. Además se cuenta con diversas maneras para revertir su efecto, entre las que se incluye la administración de vitamina $\mathrm{K}$ y de factores de la coagulación en forma de plasma fresco o como concentrado protrombínico.

Frente a ello existen numerosas desventajas, ya que el margen terapéutico es muy estrecho, tienen lento inicio de acción y requieren un estricto control, tanto por el laboratorio como por vigilancia clínica para mantener el INR en rango terapéutico a largo plazo, ya que este puede variar por numerosos factores, entre los que se incluyen la interacción con diversos alimentos y una gran cantidad de medicamentos ${ }^{5}$.

Además, la respuesta individual es variable, ya que depende de polimorfismos, como del Vitamin $K$ epoxide reductase complex subunit 1 (VKORC1), que explican hasta el $40 \%$ de la variabilidad en la dosis requerida, y del citocromo CYP2C9, que causa hasta el $10 \%$ de la variación individual ${ }^{10}$.

Al decidir un tratamiento anticoagulante con AVK se debe considerar si existe dificultad para mantener el INR en niveles óptimos, ya que numerosos factores pueden incrementarlo o disminuirlo. Los pacientes anticoagulados deben tener cuidados especiales para evitar traumatismos, limitarse en la práctica de algunos deportes y otras actividades de riesgo; incluso se debe tener precaución en la selección de otros medicamentos que pueden interferir con la respuesta a los mismos (ciprofloxacino, fluconazol, metronidazol, isoniazida, amiodarona, diltiazem, propafenona, propranolol, piroxicam, entre otros $)^{10}$. En relación con un paciente no anticoagulado, el individuo que ingiere
AVK se encuentra en mayor riesgo cuando requiere cirugías electivas o de urgencia, o cuando presenta enfermedades intercurrentes, infecciones o embarazo.

\section{Generalidades de los nuevos anticoagulantes orales}

Algunos ensayos clínicos han demostrado que los nuevos $A O D$, como apixabán, dabigatrán y rivaroxabán, son una alternativa al tratamiento convencional con AVK en la profilaxis de eventos cardioembólicos en pacientes con FANV ${ }^{11}$. Fueron creados ante la necesidad de contar con un medicamento oral que no requiriera la monitorización de su efecto, se pudiera tomar en dosis fijas y pudiera superar las limitaciones de los anticoagulantes convencionales ${ }^{12,13}$. Los AOD pueden vencer las limitaciones de los AVK, como el lento inicio de acción, la necesidad de monitorización frecuente del INR, la ventana terapéutica estrecha, las marcadas variaciones del metabolismo del medicamento inter e intrapacientes y las múltiples interacciones con medicamentos y alimentos, las cuales incrementan el riesgo de sangrado o la falta de efecto ${ }^{4}$.

A diferencia de la warfarina, que bloquea la síntesis de múltiples factores de la cascada de la coagulación (Ila, VIla, IXa, Xa), los AOD actúan específicamente inhibiendo un solo factor de la coagulación ${ }^{14}$. Según su mecanismo de acción se dividen en 2 grandes clases: los inhibidores directos de la trombina, representados por el dabigatrán, y los inhibidores del factor $X$ activado, apixabán y rivaroxabán ${ }^{15}$. Cada uno de ellos tiene una ventana terapéutica más amplia, un inicio de acción más rápido, permiten el uso de dosis fijas sin necesidad de monitorización y, comparados con la warfarina, tienen menos interacciones alimentarias o medicamentosas ${ }^{16}$.

\section{Farmacocinética, farmacodinámica y mecanismo de acción}

\section{Dabigatrán}

El dabigatrán es un inhibidor oral directo de la trombina, el cual presenta una alta afinidad con uniones reversibles ${ }^{17}$. El etexilato de dabigatrán es un profármaco que se convierte rápidamente a dabigatrán, su forma activa, mediante esterasas séricas ${ }^{18}$. Tiene una baja biodisponibilidad ( 3 a $7 \%$ ) y requiere un $\mathrm{pH}$ ácido para su absorción. Es un sustrato de la glicoproteína-P (Gp-P), un transportador de flujo, por lo que la coadministración con inductores o inhibidores de la Gp-P puede afectar su concentración plasmática ${ }^{19}$.

El pico plasmático se consigue a las $2 \mathrm{~h}$ de su administración y su vida media es de $8 \mathrm{~h}$ tras una dosis única y de 12 a $17 \mathrm{~h}$ después de múltiples dosis ${ }^{5,20}$. El porcentaje de unión a proteínas es de aproximadamente el $35 \%^{20}$. Se puede administrar con alimentos o agua para minimizar la dispepsia, aunque los alimentos no afectan la biodisponibilidad ${ }^{21}$.

El metabolismo es predominantemente renal $(80 \%)$ y es el único AOD que se puede hemodializar ${ }^{2,18}$. Su metabolismo es hepático, pero este es independiente del citocromo P450. Para el tratamiento de la FANV existen 2 presentaciones (110 y $150 \mathrm{mg}$ ) que se administran 2 veces al día (tabla 1$)^{17}$. 
Tabla 1 Tabla comparativa de la farmacocinética y farmacodinamia de los nuevos anticoagulantes orales

\begin{tabular}{llll}
\hline & Apixabán & Dabigatrán & Rivaroxabán \\
\hline Mecanismo de acción & Inh. Xa & Inh. trombina & Inh. Xa \\
Biodisponibilidad & $50 \%$ & $6 \%$ & $60 \mathrm{a} 80 \%$ \\
Concentración máxima & $3 \mathrm{~h}$ & $3 \mathrm{~h}$ & $3 \mathrm{~h}$ \\
Vida media & $9 \mathrm{a} 14 \mathrm{~h}$ & $12 \mathrm{a} 17 \mathrm{~h}$ & $5 \mathrm{a} 9 \mathrm{~h}$ \\
Excreción & $25 \%$ renal & $80 \%$ renal & $2 / 3 \mathrm{hepática}$ \\
& $75 \%$ fecal & & $1 / 3$ renal \\
Metabolismo hepático & CYP3A4 & No & $15 \mathrm{~A} 4$ \\
Dosis diaria & 2.5 a $5 \mathrm{mg}$ & 110 a $150 \mathrm{mg}$ \\
Posología & Dos veces al día & Dos veces al día & Una vez al día \\
Unión a proteínas & $90 \%$ & $35 \%$ & $90 \%$ \\
\hline
\end{tabular}

Modificado de Thachil ${ }^{21}$.

\section{Rivaroxabán}

El rivaroxabán es un inhibidor oral directo del factor $X$ activado (libre y unido al complejo protrombinasa). Es un sustrato de la Gp-P. El inicio de acción es a las $3 \mathrm{~h}$ (concentración máxima) y su vida media es de $5 \mathrm{a} 9 \mathrm{~h}$ en adultos jóvenes y de 11 a $13 \mathrm{~h}$ en adultos mayores. Tiene una biodisponibilidad del 80 al $100 \%$ con dosis de 15 a $20 \mathrm{mg}$ si se ingiere con alimentos, ya que aumenta la concentración plasmática en un 39\%; su absorción no se afecta con el $\mathrm{pH}$. Se une a las proteínas en un $90 \%$. Su metabolismo es hepático (66\%) dependiente del citocromo P450 3A4 (CYP3A4) y posteriormente su excreción es renal (33\%). Para la FA existen 2 presentaciones $(15$ y $20 \mathrm{mg}$ ) y se administra una vez al día (tabla 1) ${ }^{16,17,20}$.

\section{Apixabán \\ El apixabán es un inhibidor oral reversible y selectivo que se une al sitio activo del factor Xa. Al igual que el rivaroxa- bán, inhibe tanto al factor Xa libre como al que se encuentra unido al complejo protrombinasa. Tiene una biodisponibili- dad del $50 \%$ y es un sustrato de la Gp-P. El inicio de acción es de $3 \mathrm{~h}$ posteriores a su administración y su vida media es de 9 a $14 \mathrm{~h}$. Su metabolismo es hepático dependiente del citocromo CYP3A4 y posteriormente su excreción es renal (25\%) y el resto por las heces. Presenta unión a las proteínas del $90 \%$. Se puede administrar con o sin alimentos. Existen 2 presentaciones $(2.5$ y $5 \mathrm{mg}$ ) y se administran 2 veces al día $(\text { tabla } 1)^{5,17,20}$.}

\section{Interacciones farmacológicas}

Los AOD son metabolizados por el sistema de la Gp-P y/o el sistema citocromo P450. El citocromo P450 es un sistema oxidativo dependiente de hierro localizado primordialmente en el retículo sarcoplásmico del hígado; el sistema de la Gp-P se encuentra involucrado en el transporte de múltiples fármacos. Dado que los $3 A O D$ son un sustrato de la $\mathrm{Gp}-\mathrm{P}$, las concentraciones plasmáticas aumentan o disminuyen con la presencia de inhibidores o agonistas de la Gp-P, respectivamente $^{15}$. Los inhibidores de la Gp-P como ketoconazol, verapamilo, amiodarona, dronedarona, quinidina y claritromicina aumentan las concentraciones plasmáticas de los AOD; en cambio, inductores de la Gp-P como fenitoína, carbamazepina, rifampicina y la hierba de San Juan disminuyen los niveles de los AOD a rangos subóptimos y, por lo tanto, su coadministración debe evitarse (tabla 2$)^{2}$.

El rivaroxabán está contraindicado en combinación con fármacos que inhiben fuertemente tanto al CYP3A4 como a laGp-P como los azoles (ketoconazol, itraconazol, voriconazol, posaconazol, claritromicina y quinidina) y los inhibidores de la proteasa de VIH, ya que aumentan sus concentraciones plasmáticas. Los fármacos inductores fuertes del CYP3A4 como la rifampicina, la carbamazepina, el fenobarbital y la hierba de San Juan disminuyen su concentración plasmática. El uso concomitante con atorvastatina, digoxina o midazolam no ha demostrado generar interacciones clínicamente relevantes ${ }^{2,10}$.

En cuanto al apixabán, se debe reducir la dosis a $2.5 \mathrm{mg}$ 2 veces al día si se utiliza con inhibidores duales de la CYP3A4 y Gp-P, y debe ser evitado en pacientes que se encuentran tomando dosis reducidas de los mismos. El uso concomitante con inductores de estas vías también debe evitarse ${ }^{14}$. En la tabla 2 se presentan las interacciones farmacológicas de los AOD.

La coadministración del dabigatrán con inhibidores de bomba de protones (IBP) o bloqueadores $\mathrm{H}_{2}$ provoca reducciones pequeñas en la biodisponibilidad, pero sin alteración en la eficacia; por lo tanto, no constituye una contraindicación.

La asociación de los AOD con otros anticoagulantes, antiagregantes plaquetarios (ácido acetilsalicílico [AAS], clopidogrel, ticlopidina, prasugrel, ticagrelor, entre otros) y de antiinflamatorios no esteroideos (AINE) aumenta el riesgo de sangrado, el cual puede llegar a ser del $60 \%$ cuando se asocia con agentes antiplaquetarios ${ }^{22}$.

\section{Eventos adversos}

Los eventos adversos más frecuentes son el sangrado y las alteraciones gastrointestinales (dispepsia, gastritis, esofagitis). Comparada con warfarina la tasa de hemorragia mayor fue similar con dabigatrán $150 \mathrm{mg}(\mathrm{p}=0.32)$ y rivaroxabán $(p=0.58)$, y fue significativamente menor con apixabán $(p<0.001)$ y dabigatrán $110 \mathrm{mg}(p=0.003)$. Rivaroxabán presenta menos riesgo de hemorragia intracraneal; sin embargo, se asocia a mayor hemorragia gastrointestinal. La tasa anual de hemorragia gastrointestinal fue similar con apixabán y warfarina $(p=0.37)$. En cuanto a las alteraciones 
Tabla 2 Comparativo de interacciones farmacológicas de los nuevos anticoagulantes orales

\begin{tabular}{|c|c|c|c|c|}
\hline Fármaco & $\begin{array}{l}\text { Mecanismo } \\
\text { de interacción }\end{array}$ & Medicamentos & Efecto & Recomendaciones \\
\hline \multirow[t]{3}{*}{ Dabigatrán } & Inductor Gp-P1 & Rifampicina & Disminuye su acción & $\begin{array}{l}\text { Considerar otro } \\
\text { anticoagulante }\end{array}$ \\
\hline & $\begin{array}{l}\text { Inhibición Gp-P1 } \\
\text { (depuración de cr } \\
30-50 \mathrm{ml} / \mathrm{min} \text { ) }\end{array}$ & Ketoconazol, dronedarona & Aumenta su acción & $\begin{array}{l}\text { Reducir dosis a } 75 \mathrm{mg} \\
2 \text { veces al día o } \\
\text { considerar otro } \\
\text { anticoagulante }\end{array}$ \\
\hline & $\begin{array}{l}\text { Inhibición Gp-P1 } \\
\text { (depuración de cr } \\
\text { 15-30 ml/min) }\end{array}$ & $\begin{array}{l}\text { Ketoconazol, dronedarona, } \\
\text { amiodarona, verapamilo, } \\
\text { diltiazem, claritromicina }\end{array}$ & Aumenta su acción & $\begin{array}{l}\text { Considerar otro } \\
\text { anticoagulante }\end{array}$ \\
\hline \multirow[t]{3}{*}{ Rivaroxabán } & $\begin{array}{l}\text { Inducción fuerte } \\
\text { de CYP3A4 } \\
\text { Gp-P1 }\end{array}$ & $\begin{array}{l}\text { Rifampicina, carbamazepina, } \\
\text { fenitoína, hierba de San Juan }\end{array}$ & Disminuye su acción & $\begin{array}{l}\text { Considerar otro } \\
\text { anticoagulante }\end{array}$ \\
\hline & $\begin{array}{l}\text { Inhibición fuerte } \\
\text { de CYP3A4 } \\
\text { Gp-P1 }\end{array}$ & $\begin{array}{l}\text { Ketoconazol, itraconazol, } \\
\text { inhibidores de la proteasa } \\
\text { de VIH, conivaptán }\end{array}$ & Aumenta su acción & $\begin{array}{l}\text { Considerar otro } \\
\text { anticoagulante }\end{array}$ \\
\hline & $\begin{array}{l}\text { Inhibición leve o } \\
\text { moderada de CYP3A4 } \\
\text { Gp-P1 (depuración de } \\
\text { cr } 15-50 \mathrm{ml} / \mathrm{min})\end{array}$ & $\begin{array}{l}\text { Amiodarona, verapamilo, } \\
\text { diltiazem, eritromicina, } \\
\text { cloranfenicol, cimetidina }\end{array}$ & Aumenta su acción & $\begin{array}{l}\text { Considerar otro } \\
\text { anticoagulante }\end{array}$ \\
\hline \multirow[t]{2}{*}{ Apixabán } & $\begin{array}{l}\text { Inducción fuerte } \\
\text { de CYP3A4 } \\
\text { Gp-P1 }\end{array}$ & $\begin{array}{l}\text { Rifampicina, carbamazepina, } \\
\text { fenitoína, hierba de San Juan }\end{array}$ & Disminuye su acción & $\begin{array}{l}\text { Considerar otro } \\
\text { anticoagulante }\end{array}$ \\
\hline & $\begin{array}{l}\text { Inhibición fuerte } \\
\text { de CYP3A4 } \\
\text { Gp-P1 }\end{array}$ & $\begin{array}{l}\text { Ketoconazol, itraconazol, } \\
\text { inhibidores de la proteasa } \\
\text { de VIH, claritromicina }\end{array}$ & Aumenta su acción & $\begin{array}{l}\text { Reducir la dosis a } \\
2.5 \mathrm{mg} 2 \text { veces al día } \\
\text { o considerar otro } \\
\text { anticoagulante }\end{array}$ \\
\hline
\end{tabular}

cr: creatinina; Gp-P1: glicoproteína-P1, VIH: virus de inmunodeficiencia humana.

Modificado de Tanaka-Esposito y Chung ${ }^{16}$.

gastrointestinales, estas fueron mayores con warfarina que con dabigatrán $(p<0.001)$ en ambas dosis ${ }^{14,19}$.

\section{Importancia de la adherencia}

Los AOD tienen un efecto anticoagulante muy predecible, y la monitorización del efecto anticoagulante no está indicada como con los AVK (a menos que cursen con enfermedades específicas, como la enfermedad renal crónica [ERC]). Sin embargo, el efecto se pierde rápidamente después de 12 a $24 \mathrm{~h}$ posteriores a la última dosis; por tal motivo, la adherencia estricta al tratamiento es fundamental. Se sabe que el apego al tratamiento en promedio es $<80 \%$. Por eso es importante tener estrategias para lograr un mayor apego al tratamiento anticoagulante (tabla 3$)^{23}$.

La dosis de una vez al día se ha relacionado con mayor adherencia comparada con la dosis de 2 veces al día en pacientes con patología cardiovascular y en FA (en el caso de medicamentos para diabetes mellitus [DM] e hipertensión arterial [HTA]). Es probable que para los AOD la dosis de una vez al día tenga la misma adherencia, aunque realmente se desconoce si alguna de estas 2 opciones es superior para prevenir un evento trombótico o de sangrado como en los estudios clínicos ${ }^{23}$.

\section{Errores de dosificación}

Una ventaja o desventaja de los AOD ante los AVK es la vida media: mientras que para la warfarina es de 48 a $72 \mathrm{~h}$, para los AOD es de 10 a $12 \mathrm{~h}$ en promedio (excepto para rivaroxabán, que en pacientes jóvenes es de 5 a $6 \mathrm{~h}$ ). La vida media de los nuevos anticoagulantes requiere un apego estricto a la prescripción del medicamento; la falta de toma de una dosis o el retraso en la misma pueden exponer al paciente a presentar un evento tromboembólico central o periférico, particularmente con los medicamentos con dosis de una vez al día ${ }^{23}$.

Dado que los AOD tienen una vida media corta, es de suma importancia el apego al tratamiento ${ }^{23}$. Para este efecto los AOD se dividen según su posología, y los errores de dosificación se esquematizan en la tabla 4.

\section{La descontinuación de los anticoagulantes orales directos a largo plazo}

La falta de adherencia es más frecuente en el tratamiento de enfermedades crónicas que en las terapias a corto plazo por padecimientos agudos. Los factores asociados a la baja adherencia identificados en diversos estudios incluyen la frecuencia de administración, problemas sociales, la deficiente 
Tabla 3 Consideraciones prácticas para mejorar el apego al tratamiento de los nuevos anticoagulantes orales

La educación del paciente en la estricta adherencia es el objetivo de mayor importancia. Deben buscarse las herramientas adecuadas como la información en el consultorio, llevar un registro de las tomas del medicamento, grupos de apoyo, alarmas en dispositivos inteligentes, etc.

Los miembros de la familia deben involucrarse de la misma manera usando estas herramientas. Les debe quedar clara la importancia del correcto uso de los medicamentos en su familiar enfermo

Dejar en claro que la monitorización por laboratorio no será necesaria con los AOD

Diseño de herramientas adecuadas para el recordatorio de la toma de los medicamentos mediante llamadas telefónicas, alarmas de relojes, uso de dispositivos inteligentes

Reconsiderar a los pacientes que prefieren la monitorización de la anticoagulación con el uso del INR A los pacientes con mala adherencia al tratamiento a los AOD se les debe tratar con AVK por su larga vida media

AOD: anticoagulantes orales directos; AVK: antagonistas de la vitamina K; INR: índice internacional normalizado.

Modificado de Heidbuchel et al. ${ }^{23}$.

Tabla 4 Errores de dosificación

\begin{tabular}{|c|c|c|c|c|}
\hline $\begin{array}{l}\text { Esquema de } \\
\text { dosificación }\end{array}$ & Dosis faltante & Dosis doble & $\begin{array}{l}\text { Incertidumbre } \\
\text { respecto a la dosis }\end{array}$ & Sobredosis \\
\hline $\begin{array}{l}\text { Dos veces al día } \\
\text { (dabigatrán, } \\
\text { apixabán) }\end{array}$ & $\begin{array}{l}\text { Hasta } 6 \text { h después } \\
\text { de la dosis }\end{array}$ & $\begin{array}{l}\text { Omitir la siguiente } \\
\text { dosis }\end{array}$ & $\begin{array}{l}\text { No tomar otra dosis; } \\
\text { continuar normal }\end{array}$ & $\begin{array}{l}\text { Dependiendo de la cantidad de } \\
\text { sobredosis sospechada, se sugieren } \\
\text { tomar medidas como la }\end{array}$ \\
\hline $\begin{array}{l}\text { Una vez al día } \\
\text { (rivaroxabán) }\end{array}$ & $\begin{array}{l}\text { Hasta } 12 \mathrm{~h} \text { después } \\
\text { de la dosis }\end{array}$ & $\begin{array}{l}\text { Continuar la siguiente } \\
\text { dosis normal }\end{array}$ & $\begin{array}{l}\text { Tomar otra dosis; } \\
\text { continuar normal }\end{array}$ & $\begin{array}{l}\text { hospitalización para monitorización } \\
\text { o medidas urgentes }\end{array}$ \\
\hline
\end{tabular}

Modificado de Heidbuchel et al. ${ }^{23}$.

relación médico-paciente, el nivel educacional, el número de dosis, el estado ocupacional y la discapacidad. Algunos estudios han reportado que dosis más altas son necesarias para alcanzar niveles de eficacia de anticoagulación similares para el mismo medicamento en pacientes con mala adherencia a los $\mathrm{AOD}^{24}$.

Los AOD con esquemas de 2 veces al día como dabigatrán y apixabán tienen mayores tasas de no adherencia debido a que la frecuencia de su administración es superior ${ }^{24}$.

Sin embargo, un metaanálisis de 18 estudios (101,801 pacientes) evaluó la descontinuación de los AOD a largo plazo. Los pacientes tenían varias indicaciones para anticoagulación a largo plazo: tromboembolia venosa, pulmonar, prevención de EVC en pacientes con FANV y pacientes con síndrome coronario agudo. Dentro de los resultados se observó que no hay diferencia significativa en el abandono del tratamiento entre los AOD, independientemente de la dosis usada, y la warfarina en todos los escenarios, a excepción del tratamiento del síndrome coronario agudo, donde el abandono fue mayor que con el grupo control ${ }^{24}$.

\section{Seguimiento}

El seguimiento es de suma importancia, particularmente por tratarse de una población vulnerable con posibilidades de sufrir un evento vascular isquémico o una hemorragia. Los AOD, aunque tienen pocas interacciones con otros medicamentos, no están libres de las mismas y de tener complicaciones, por lo que se recomienda un adecuado control y seguimiento Para tal efecto, en el Anexo 3 se muestra un ejemplo de tarjeta de control de AOD.

\section{Intercambio de terapia: AVK-AOD, AOD-AVK, AOD-AOD}

\section{Cambio de terapia anticoagulante}

Es importante asegurar la continuidad del tratamiento anticoagulante mientras se minimiza el riesgo de hemorragia cuando se hace el cambio entre las diferentes terapias. Para hacer el cambio es necesario tener el conocimiento de la farmacocinética y la farmacodinamia de los diferentes anticoagulantes a utilizar, ya que es importante individualizar la decisión para cada paciente ${ }^{23}$.

\section{Cambio de los antagonistas de la vitamina $\mathrm{K}$ a anticoagulantes orales directos}

Los AOD pueden iniciarse inmediatamente cuando un paciente que esté tomando antagonistas de la vitamina $K$ tenga un INR menor de 2; si el INR es de 2.0 a 2.5 los AOD pueden iniciarse al día siguiente. Cuando el INR es mayor de 2.5 debe tenerse en cuenta la vida media de los anticoagulantes antes de iniciar la terapia con AOD: la vida media de la acenocumarina es de 8 a $14 \mathrm{~h}$, mientras que de la warfarina es de 36 a $42 h^{23}$.

\section{Cambio de anticoagulación intravenosa a anticoagulantes orales directos}

Cuando algún paciente esté recibiendo heparina no fraccionada (HNF) intravenosa, los AOD se pueden iniciar una vez que esta sea interrumpida, recordando que la vida media 
de la HNF es de $\pm 2 \mathrm{~h}$, prestando atención a los pacientes que tengan datos de ERC, en los que generalmente su eliminación suele tomar más tiempo. En los pacientes que estén recibiendo heparina de bajo peso molecular (HBPM), generalmente los AOD pueden iniciarse cuando esté prevista la siguiente dosis de HBPM, tomando en cuenta el AOD a utilizarse $^{23}$

\section{Cambio de los anticoagulantes orales directos hacia antagonistas de la vitamina $\mathrm{K}$}

El inicio de acción de los AVK puede tardar hasta 5 a 10 días antes de que se obtenga un INR en rango terapéutico, y ello obedece a una amplia variabilidad en cada paciente; por lo tanto, ambos deben administrarse de manera concomitante hasta obtener un INR en rango terapéutico ${ }^{23}$.

No es recomendable usar dosis de impregnación para acenocumarina y/o warfarina, ya que los AOD pueden aumentar el valor del INR, especialmente los inhibidores del factor Xa. Durante el traslape debe determinarse el INR antes de recibir la siguiente dosis del AOD y nuevamente $24 \mathrm{~h}$ después de la última dosis de AOD para estar seguro de tener buena anticoagulación. Es recomendable una monitorización continua del INR dentro del primer mes hasta obtener valores estables entre 2.0 y $3.0^{23}$.

\section{Cambio de anticoagulantes orales directos hacia anticoagulación intravenosa}

Debido al metabolismo de la HNF y la HBPM, estos anticoagulantes pueden iniciarse cuando corresponda la siguiente dosis del $A O D^{23}$.

\section{Cambio de un anticoagulante oral directo a otro}

Los AOD pueden iniciarse cuando le corresponda la siguiente dosis del AOD; sin embargo, debe tenerse cuidado en los pacientes que pueden tener altas concentraciones plasmáticas del medicamento, como es el caso de pacientes con ERC (en los que es recomendable tener un intervalo más largo en la siguiente dosis) y considerar siempre la depuración de creatinina $(\mathrm{DCr})^{23}$.

\section{Estratificación del riesgo}

\section{Estratificación del riesgo de evento vascular cerebral isquémico}

El EVC es una de las complicaciones más importantes que puede sufrir un paciente con FA, y se tiene estimado que sin tromboprofilaxis este riesgo aumenta hasta 7 veces por encima del que tiene la población general; los EVC relacionados con FANV tienen mayor recurrencia y morbimortalidad si se comparan con los EVC no relacionados con $\mathrm{FA}^{25}$.

Se han identificado varios factores de riesgo asociados con un incremento en la ocurrencia de un EVC de tipo isquémico. Las características clínicas que de manera más consistente han sido relacionadas son: el antecedente de un EVC o ataque isquémico transitorio (AIT), HTA, DM y edad. Otros factores son la disfunción ventricular izquierda, la enfermedad aterosclerosa de la aorta (placa observada por ecocardiograma transesofágico), la EAP, y también hay evidencia de que las mujeres tienen un riesgo mayor que los hombres ${ }^{26}$.

Tomando en cuenta los factores clínicos antes mencionados, se han estudiado diversos modelos para cuantificar el riesgo de EVC en pacientes con FA y para orientar las decisiones de tratamiento preventivo ${ }^{25}$. La escala $\mathrm{CHADS}_{2}$ estima el riesgo basándose en la presencia de insuficiencia cardiaca congestiva (ICC), HTA, edad $\geq 75$ años, DM, antecedente de EVC o AIT o tromboembolismo (Anexo 1). Esta escala ha sido validada en múltiples estudios que indican una tasa aproximada del $2 \%$ de aumento de riesgo por cada punto que incrementa el $\mathrm{CHADS}_{2}$. Una limitación es que una puntuación de 1 se considera como riesgo intermedio; por lo tanto, aquellos con riesgo más bajo no están bien identificados. También pacientes con puntuación de 2 debido a antecedente de EVC tienen mayor riesgo del que indica la escala. Para contrarrestar estas limitaciones se formuló la escala $\mathrm{CHA}_{2} \mathrm{DS}_{2}$-Vasc (Anexo 2), la cual toma en cuenta, además de las variables incluidas en la $\mathrm{CHADS}_{2}$, los factores de enfermedad vascular, la edad entre 65 y 74 años y el género. Cuando se compararon ambas escalas, se observó que el $\mathrm{CHA}_{2} \mathrm{DS}_{2}$. Vasc es capaz de discriminar mejor a los pacientes con $\mathrm{CHADS}_{2}$ de 0 a 1, logrando una mejor habilidad predictiva, y ha definido recomendaciones de anticoagulación más claras. Por tal motivo, actualmente las guías recomiendan el uso de la escala $\mathrm{CHA}_{2} \mathrm{DS}_{2}$-Vasc para cuantificar el riesgo de EVC ${ }^{2}$.

La estratificación del riesgo ayuda a tomar la decisión terapéutica en lo que respecta al tratamiento antitrombótico. En términos generales, las guías recomiendan que los pacientes con FANV y un $\mathrm{CHA}_{2} \mathrm{DS}_{2}$-Vasc de 0 se mantengan sin tratamiento antitrombótico; con un $\mathrm{CHA}_{2} \mathrm{DS}_{2}$-Vasc de 1 tampoco se recomienda anticoagulación o se puede considerar tratamiento con $\mathrm{AO}$ o AAS; con un antecedente de EVC, TIA o $\mathrm{CHA}_{2} \mathrm{DS}_{2}$-Vasc $\geq 2$ se recomienda la anticoagulación con warfarina, apixabán, dabigatrán o rivaroxabán. En pacientes con ERC moderada a severa y $\mathrm{CHA}_{2} \mathrm{DS}_{2}-\mathrm{Vasc} \geq 2$ se pueden considerar dosis bajas de $\mathrm{AOD}^{2}$.

\section{Estratificación del riesgo de hemorragia}

La hemorragia mayor se convierte en un riesgo adicional propio del tratamiento anticoagulante en pacientes con FA, particularmente la hemorragia intracerebral $(\mathrm{HIC})$, siendo esta la principal controversia en el tratamiento entre el riesgo de trombosis y hemorragia. Aunque la HIC es la complicación más grave y mortal, también las hemorragias se pueden localizar en el espacio retroperitoneal, intraespinal, pericárdico e intraarticular ${ }^{1,27}$.

La incidencia anual de hemorragia mayor en los diferentes estudios que comparan la warfarina con los AOD se reporta en rangos que varían entre 3.09 a 3.4 y de 2.13 a 3.6 , respectivamente (tabla 5$)^{1}$. Además, se calcula que la tasa de HIC se ha quintuplicado en los años recientes en relación con el aumento en el uso de fármacos antiplaquetarios y anticoagulantes en adultos mayores ${ }^{27}$.

Las escalas para cuantificar el riesgo de hemorragia mayor (hemorragia que involucra sitios anatómicos críticos como intracraneal, que requiera hospitalización, 
Tabla 5 Comparativo de incidencia anual de hemorragia con los nuevos anticoagulantes orales

\begin{tabular}{|c|c|c|c|c|c|c|c|}
\hline & \multicolumn{3}{|c|}{ RE-LY (dabigatrán) } & \multicolumn{2}{|c|}{ ROCKET-AF (rivaroxabán) } & \multicolumn{2}{|c|}{ ARISTOTLE (apixabán) } \\
\hline & \multicolumn{7}{|c|}{ Resultados (\% anual) } \\
\hline & $\begin{array}{l}\text { Warfarina } \\
n=6,022\end{array}$ & $\begin{array}{l}\text { D } 150 \\
n=6,076 \\
(R R, I C 95 \%, p)\end{array}$ & $\begin{array}{l}\text { D } 110 \\
n=6,015 \\
(R R, I C 95 \%, p)\end{array}$ & $\begin{array}{l}\text { Warfarina } \\
n=7,133\end{array}$ & $\begin{array}{l}\text { Rivaroxabán } \\
\mathrm{n}=7,131 \\
(\mathrm{HR}, \mathrm{IC} 95 \%, \mathrm{p})\end{array}$ & $\begin{array}{l}\text { Warfarina } \\
n=9,081\end{array}$ & $\begin{array}{l}\text { Apixabán } \\
n=9,120 \\
(H R, I C 95 \%, p)\end{array}$ \\
\hline Hemorragia mayor & 3.36 & $\begin{array}{l}3.11(0.93 \\
0.81-1.07 \\
p=0.31)\end{array}$ & $\begin{array}{l}2.71(0.8 \\
0.69-0.93 \\
p=0.003)\end{array}$ & 3.4 & $3.6(p=0.58)$ & 3.09 & $\begin{array}{l}2.13(0.69 \\
0.6-0.8 \\
p<0.001)\end{array}$ \\
\hline $\begin{array}{l}\text { Hemorragia } \\
\text { intracraneal }\end{array}$ & 0.74 & $\begin{array}{l}0.3(0.4 \\
0.27-0.6 \\
p<0.001)\end{array}$ & $\begin{array}{l}0.23(0.31 \\
0.2-0.47 \\
p<0.001)\end{array}$ & 0.7 & $\begin{array}{l}0.5(0.67 \\
0.47-0.93 \\
p=0.02)\end{array}$ & 0.8 & $\begin{array}{l}0.33(0.42 \\
0.3-0.58 \\
p<0.001)\end{array}$ \\
\hline $\begin{array}{l}\text { Hemorragia } \\
\text { extracraneal }\end{array}$ & 2.67 & $\begin{array}{l}2.84(1.07 \\
0.92-1.25 \\
p=0.38)\end{array}$ & $\begin{array}{l}2.51(0.94 \\
0.8-1.1 \\
p=0.45)\end{array}$ & & & & \\
\hline EVC hemorrágico & 1.2 & $\begin{array}{l}0.92(0.76 \\
0.6-0.98 \\
p<0.03)\end{array}$ & $\begin{array}{l}1.34(1.11 \\
0.89-1.4 \\
p<0.35)\end{array}$ & 1.42 & $\begin{array}{l}1.34(0.94 \\
0.75-1.17 \\
p=0.581)\end{array}$ & 1.05 & $\begin{array}{l}0.97(0.92 \\
0.74-1.13 \\
p<0.42)\end{array}$ \\
\hline $\begin{array}{l}\text { Hemorragia } \\
\text { gastrointestinal }\end{array}$ & 1.02 & $\begin{array}{l}1.51(1.5 \\
1.19-1.89 \\
p<0.001)\end{array}$ & $\begin{array}{l}1.12(1.1 \\
0.86-1.41 \\
p=0.43)\end{array}$ & 2.2 & $3.2(p<0.001)$ & 0.86 & $\begin{array}{l}0.76(0.89 \\
0.7-1.15 \\
p=0.37)\end{array}$ \\
\hline
\end{tabular}

EVC: evento vascular cerebral.

Modificado de Camm et al. ${ }^{52}$.

transfusión de $\geq 2$ unidades o paquetes celulares, o una disminución de $\geq 2 \mathrm{~g} /$ de hemoglobina) incluyen la HAS-BLED (HTA, función renal o hepática anormal, EVC, antecedente o predisposición de sangrado, INR lábil, edad avanzada, uso concomitante de alcohol o drogas), la RIETE (anemia, edad $>75$ años, cáncer o embolia pulmonar), la HEMORR 2 hages (enfermedad hepática o renal, abuso de etanol, cáncer, edad avanzada, trombocitopenia, resangrado, HTA, anemia, factores genéticos, riesgo excesivo de caídas o de EVC) y la ATRIA (anemia, IRC, edad > 75 años, hemorragia previa, HTA $)^{2,25}$.

La escala HAS-BLED es una escala práctica validada para la estimación del riesgo de hemorragia y se considera mejor que los sistemas de puntuación de $\mathrm{HEMORR}_{2}$ hages y ATRIA para discriminar el riesgo, aunque todas presentan un rendimiento modesto y baja precisión predictiva (Anexo 4$)^{16}$. Por lo tanto, no debe utilizarse para tomar decisiones acerca de la anticoagulación de un paciente, por ejemplo para excluirlos, sino que se sugiere su uso para identificar a aquellos con riesgo alto (puntuación $\geq 3$ ) en quienes se deberá vigilar de manera más estrecha y monitorizar su INR ${ }^{15}$.

\section{Manejo de la hemorragia y sus complicaciones}

Estudios muestran que, por año, aproximadamente del 0.2 al $0.5 \%$ de los pacientes con FA en tratamiento con AOD tienen como complicación una hemorragia cerebral ${ }^{26}$. Los riesgos relativos de hemorragia cambian de acuerdo a la dosis y al medicamento, y comparado con la warfarina, el riesgo de hemorragia en la mayoría de los casos es menor o comparable (tabla 5) ${ }^{1}$.

Aunque los $A O D$ no requieren monitorización, esta sí es necesaria en casos especiales como las hemorragias para asegurar que no exista sobre anticoagulación y para determinar la eficacia de los agentes que revierten su efecto ${ }^{21,25}$. Las pruebas como el tiempo de ecarina, el tiempo de trombina (TT) o el tiempo de tromboplastina parcial activado (TTPa) evalúan el efecto del dabigatrán, y el tiempo de protrombina y una prueba anti-Xa pueden evaluar el efecto de apixabán y rivaroxabán, aunque su utilidad en ambos casos es limitada 23,28 .

Debido a que no se cuenta con mediciones apropiadas de la intensidad de la anticoagulación con estos fármacos ni se dispone de antídotos específicos, la mayoría de las recomendaciones para el manejo de hemorragias derivan de consensos de expertos o de criterios de laboratorio ${ }^{23,29}$.

Los pacientes con hemorragia deben ser estratificados de acuerdo a su estabilidad hemodinámica, el sitio de sangrado y la severidad de la hemorragia. También se deben tomar en cuenta factores como la indicación de la anticoagulación, la dosis y la hora de la última toma, la función renal y las comorbilidades. Debido a las limitaciones en cuanto a la falta de agentes específicos que reviertan el efecto de los $A O D$ y la poca información acerca de terapias no específicas, las medidas de soporte y la referencia urgente se consideran los pilares del manejo de la hemorragia ${ }^{30}$.

De acuerdo a su severidad, las hemorragias se pueden clasificar en menores, que son aquellas que pueden necesitar atención médica pero que no requieren transfusión u hospitalización, suelen autolimitarse y no necesitan la suspensión de anticoagulación (solo necesaria en caso de no autolimitarse), y en hemorragias mayores, que son las que involucran sitios críticos (intracraneal, intraespinal, intraocular, retroperitoneal, intraarticular, pericárdica, intramuscular con síndrome compartimental), causan una disminución de la hemoglobina $\geq 20 \mathrm{~g} / \mathrm{l}$ en $24 \mathrm{~h}$ o requieren transfusión de más de 2 paquetes globulares ${ }^{29,30}$. Dependiendo de su severidad, 


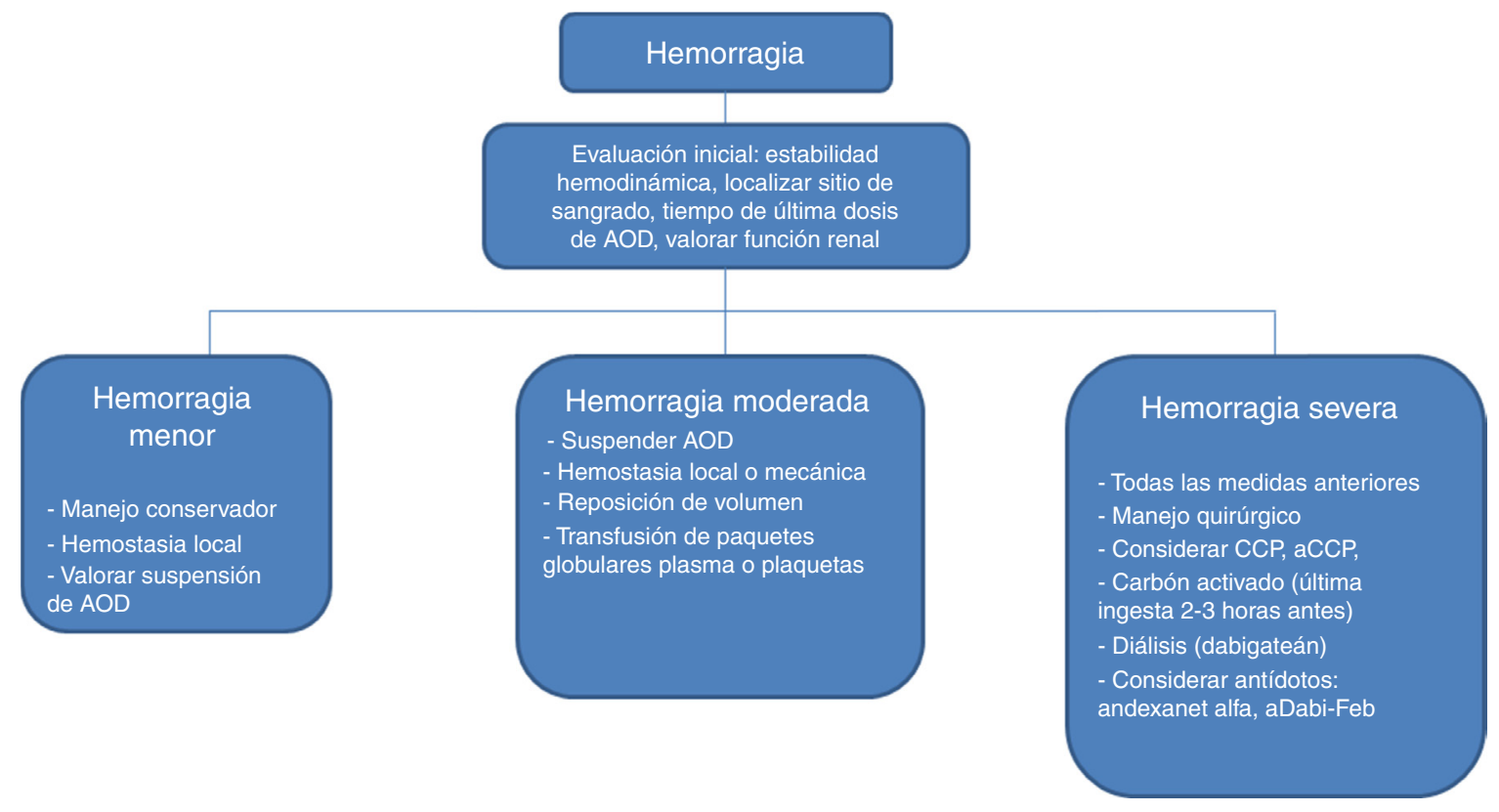

Figura 1 Tratamiento sugerido para los pacientes con hemorragia aguda en tratamiento con anticoagulantes orales directos. aCCP: concentrado de complejo de protrombínico activado; AOD: anticoagulantes orales directos; CCP: concentrado de complejo de protrombínico.

Modificado de Siegal et al. ${ }^{30}$ y Thachil $^{21}$.

se pueden tomar varias rutas de tratamiento, que incluyen una tercera categoría intermedia en la que la hemorragia es grave pero no implica sitios de alto riesgo (fig. 1$)^{30}$.

Las hemorragias menores (epistaxis, equimosis, menorragia) pueden tratarse con manejo conservador, como medidas de hemostasia local y evaluar la posibilidad de suspender al menos una dosis del anticoagulante, al poner en la balanza el riesgo de trombosis contra el de hemorragia ${ }^{29,30}$.

Las hemorragias con sangrado moderado requieren de las medidas generales, y además es necesario suspender el anticoagulante, considerando su reinicio dependiendo del estado del paciente y de las intervenciones mecánicas o locales de hemostasia. En pacientes con alto riesgo trombótico se puede considerar la aplicación de una dosis parenteral baja de anticoagulante, transfusiones de paquetes globulares, plasma o plaquetas ${ }^{30}$.

Las hemorragias severas o que amenazan la vida necesitan ser tratadas en un ámbito de cuidados intensivos, con acceso rápido a todos los medios necesarios, ya que, además del soporte hemodinámico, requieren manejo quirúrgico y el empleo de factores procoagulantes como el concentrado de complejo protrombínico (CCP) que contiene altas dosis de factores de coagulación derivados de vitamina K (II, VII, $\mathrm{IX}, \mathrm{x}$ ) inactivados y cantidades variables de proteína $\mathrm{C}$ y $\mathrm{S} 0$ del concentrado de complejo protrombínico activado (aCCP) que contiene cantidades pequeñas de estos factores en su forma activa $^{30}$.

Cuando el paciente ha ingerido la última dosis de anticoagulante en un lapso menor a 2-3 h de su llegada a un servicio médico, se puede administrar carbón activado. La hemodiálisis puede considerarse en casos de hemorragia severa por dabigatrán y en pacientes con insuficiencia renal, en quienes se puede remover hasta el 68\% del fármaco (en algunos estudios, hasta un $77 \%$ ). Apixabán y rivaroxabán no son dializables por su elevado nivel de unión a proteínas. Por otro lado, el hecho de suspender una o 2 dosis del medicamento puede ayudar a controlar la hemorragia, ya que la vida media de los AOD es más estable que las de los AVK ${ }^{2}$.

Es necesario recordar que muchos de los productos mencionados para el control de la hemorragia se desarrollaron para el tratamiento de las hemofilias, por lo que su uso en personas sin la deficiencia de los factores de la coagulación puede ocasionar trombosis. Este mismo fenómeno ocurre con el factor vı activado, que además no ha demostrado utilidad en los pacientes tratados con $\mathrm{AOD}^{30}$.

Algunos nuevos antídotos se encuentran actualmente en desarrollo y evaluación, dado el caso del andexanet alfa, un antídoto de proteína recombinante específico para los inhibidores del factor $\mathrm{Xa}$, que se encuentra en fase 2 de experimentación, reportando alta capacidad de reversibilidad para apixabán ${ }^{25,29}$. También se está desarrollando un anticuerpo monoclonal contra dabigatrán (aDabi-Fab) ${ }^{25,29}$.

No se cuenta con datos clínicos suficientes sobre el uso de plasma, hemoperfusión con carbón activado, desmopresina y otros antifibrinolíticos (ácido tranexámico o aminocaproico) en los pacientes tratados con AOD ${ }^{21,30}$.

\section{Reinicio de la anticoagulación después de hemorragia intracerebral}

El reinicio de la anticoagulación después de una HIC debe ser individualizado, considerando, por un lado, el riesgo de hemorragia recurrente $y$, por otro, el riesgo de EVC o de embolia sistémica con y sin anticoagulantes ${ }^{28}$.

El riesgo de HIC recurrente parece ser mayor en pacientes con hemorragia lobar que en hemorragias de localización en los ganglios basales. Otros predictores de recurrencia de 
HIC incluyen el antecedente de EVC isquémico, DM, uso de AAS y los predictores de riesgo de la escala HAS-BLED. En contraste, el riesgo es menor en pacientes con un adecuado control de la HTA 28 .

Se ha visto que hay menor riesgo de recurrencia de HIC en pacientes tratados con los AOD comparado con warfarina; por tal motivo, en pacientes que sufrieron una HIC durante el tratamiento a base de warfarina se sugiere reiniciar la anticoagulación con los $\mathrm{AOD}^{28}$.

Algunos estudios recomiendan que el tiempo óptimo para reiniciar la anticoagulación es entre las 10 y las 30 semanas posteriores a la HIC, aunque otros estudios sugieren que sea entre la primera a la tercera semana en caso de alto riesgo tromboembólico y bajo riesgo de $\mathrm{HIC}^{28}$.

\section{Pacientes con evento vascular cerebral isquémico agudo en tratamiento con anticoagulantes orales directos}

\section{Trombólisis en pacientes con infarto cerebral agudo tratados con anticoagulantes orales directos}

Diferentes ensayos clínicos sugieren que cada año entre el 1.0 y el $2.0 \%$ de los pacientes con FA y del 0.1 al $0.2 \%$ de los pacientes con trombosis venosa profunda que se encuentran en tratamiento con los AOD desarrollan un infarto cerebral agudo (ICA) ${ }^{28}$.

El único tratamiento que ha probado modificar la evolución natural de los pacientes con ICA es el activador tisular del plasminógeno humano (rtPA) administrado por vía intravenosa en las primeras 3 a $4.5 \mathrm{~h}$ de evolución; sin embargo, las principales complicaciones de su administración son las hemorrágicas, especialmente devastadoras cuando ocurren a nivel cerebral, como la HIC. Las guías actuales sugieren que en pacientes tratados con acenocumarina o warfarina se puede administrar rtPA, siempre y cuando se encuentren en las primeras $3 \mathrm{~h}$ de evolución y la determinación de INR sea menor a 1.7 o el tiempo de protrombina (TP) $<15 \mathrm{~s}^{31}$. Los pacientes tratados con los AOD que desarrollan un ICA no deben ser considerados para tratamiento con trombólisis intravenosa, a menos que se constate la ausencia clínica y por laboratorio (TTPa, INR, recuento plaquetario, tiempo de coagulación con eucarina, TT) de su efecto anticoagulante, o si la última dosis del medicamento fue el equivalente, en tiempo, a 2 veces la vida media del AOD (aproximadamente $24 \mathrm{~h}$ ) en pacientes con función renal normal; otros autores recomiendan que el tiempo sea de por lo menos 4 vidas medias del medicamento $(48 \mathrm{~h})^{28,31}$. Se sabe que están en desarrollo pruebas específicas para evaluar las concentraciones plasmáticas y el efecto anticoagulante. Mientras tanto, y dado que no se cuenta con agentes específicos que reviertan el efecto anticoagulante de los $A O D$, se sugiere no utilizar rtPA en pacientes con ICA en tratamiento con $\mathrm{AOD}^{28}$.

Otras alternativas para pacientes con ICA son la terapia endovascular, en la que puede utilizarse trombólisis selectiva o intraarterial, dispositivos para trombectomía mecánica o por aspiración, uso de guía o microcatéter para manipular el coágulo y angioplastia y colocación de stent $^{28,31}$.

\section{Reinicio de anticoagulación después de un infarto} cerebral agudo

El reinicio de la anticoagulación después de un ICA es un tema de amplio debate, sobre todo por el alto riesgo de transformación hemorrágica o hemorragia cerebral en infartos cerebrales de etiología embólica ${ }^{28}$.

El riesgo de recurrencia de un EVC o de ES es mayor en pacientes con FA y puntajes altos de $\mathrm{CHADS}_{2}$ y $\mathrm{CHA}_{2} \mathrm{DS}_{2}$-Vasc, disfunción ventricular izquierda evidenciada por ecocardiograma transesofágico y crecimiento o trombo en la aurícula izquierda ${ }^{28}$.

En una revisión sistemática que incluyó a 23,748 pacientes, el tratamiento con anticoagulantes en los primeros días de evolución disminuyó la frecuencia de recurrencia pero incrementó la tasa de hemorragia cerebral sintomática. Es por ello que algunas guías sugieren que en pacientes con FANV e ICA los anticoagulantes deben reiniciarse una a 2 semanas después del infarto cerebral, tiempo en el cual disminuye el riesgo de transformación hemorrágica. Los ensayos clínicos con AOD (ROCKET AF, RELLY, ARISTOTLE) solo incluyeron pacientes con ICA en este periodo de tiempo. Algunas guías de tratamiento recomiendan continuar los AOD dependiendo del tamaño del infarto cerebral, mientras que, en la práctica diaria, en muchos centros se utiliza la regla de 1-3-6-12 días: en pacientes con AIT se puede reiniciar la anticoagulación el día uno de evolución, en pacientes con infarto menor no incapacitante después de 3 días, en infartos menores de un tercio de la cerebral media después del día 6 , y en infartos extensos después de 2 a 3 semanas $^{28}$.

\section{Pacientes con cardiopatía isquémica}

\section{Uso de los nuevos anticoagulantes orales en pacientes con síndrome coronario agudo y fibrilación auricular}

Posterior a un síndrome coronario agudo (SICA), los pacientes permanecen con un riesgo significativo de presentar recurrencia de eventos tromboembólicos como infarto agudo de miocardio (IAM), isquemia, EVC o muerte en los siguientes meses ${ }^{25,32}$. Por lo tanto, se recomienda el uso de terapia antitrombótica para la prevención a largo plazo de eventos isquémicos ${ }^{33}$.

La terapia antiplaquetaria dual (TAPD), que incluye el AAS en combinación con clopidogrel, ha demostrado una reducción del $20 \%$ de riesgo de muerte, IAM y EVC después de un mes y hasta por un año, comparado con AAS como monoterapia ${ }^{33}$. Sin embargo, a pesar de la TAPD, del 9 al $11 \%$ de los pacientes con SICA continúan presentando eventos al año del episodio ${ }^{34}$.

Se ha investigado el uso de los AOD en el manejo de pacientes con SICA como aditivo a la TAPD en diversos estudios $^{35}$.

Un metaanálisis que incluyó a 31,286 pacientes y comparó el riesgo-beneficio del uso de los AOD en pacientes tratados con terapia antiplaquetaria posterior a un SICA mostró una reducción en los eventos isquémicos a costa de un aumento en el riesgo de hemorragia mayor ${ }^{32}$.

Estudios de fase ॥ y III como el RE-DEEM para dabigatrán, el ATLAS ACS TIMI-46 para rivaroxabán y el APRAISE-1 y 2 para 
apixabán han investigado la seguridad y la eficacia del uso de los AOD asociados con terapia antiplaquetaria sola (AAS) o dual en pacientes con SICA, comparados con placebo. Los resultados han demostrado un aumento significativo en el riesgo de hemorragia mayor sin mejoría clínica de los desenlaces con el uso de AOD más TAPD ${ }^{25,33,35}$. Por lo tanto, ningún $A O D$ ha sido aprobado por la FDA en este escenario; rivaroxabán es el único aprobado en Europa por la European Medicines Agency (EMA), pero continúa sin su aprobación por la FDA ${ }^{35}$. Las guías de la AHA/ACC/HRS tampoco recomiendan su uso ${ }^{2}$.

\section{Manejo del síndrome coronario agudo en pacientes con fibrilación auricular en tratamiento con anticoagulantes orales directos}

En el momento que un paciente con FA tratado con $A O D$ presenta un SICA es recomendable interrumpirlo temporalmente. Se debe iniciar una TAPD a menos de que el paciente presente alto riesgo de hemorragia. En estos pacientes se sugiere utilizar AAS hasta que se termine el efecto del AOD y proseguir con la TAPD. Se sugiere como tratamiento estándar administrar dosis bajas de AAS a su ingreso (150 a $300 \mathrm{mg}$ de carga; 75 a $100 \mathrm{mg}$ posteriormente) y combinarlo con inhibidores de los receptores de ADP (preferir ticagrelor o prasugrel sobre clopidogrel). Posteriormente, se debe iniciar anticoagulación parenteral con fondaparinux en el caso de SICA sin elevación del segmento ST (SICASEST) ${ }^{23}$.

En el caso de que se trate de un IAM con elevación del segmento ST, se prefiere la intervención coronaria percutánea (ICP) primaria sobre la fibrinólisis. Si esta última es la única disponible, se debe evitar el uso de HNF o enoxaparina hasta que desaparezca el efecto de los $A O D^{23}$.

Para el SICASEST debe evaluarse la urgencia; de no haberla, es recomendable retrasar la angiografía coronaria hasta que disminuya el efecto del AOD. Se debe iniciar la terapia de anticoagulación en la fase periprocedimiento con $\mathrm{HNF}^{23}$.

En la ICP se prefiere el abordaje radial sobre el femoral. Si está indicado se puede realizar angioplastia con balón evitando el uso de stent, aunque si es requerido se deben utilizar stents metálicos no recubiertos (bare metal stent), utilizando anticoagulación parenteral adicional al tratamiento integral. Se recomienda la bivalirudina periprocedimiento, suspendiéndola tras la ICP ${ }^{23}$.

Se sugiere la cirugía de bypass en los pacientes que requieren terapia de revascularización extensa ${ }^{23}$.

Al reiniciar un AOD hay que considerar la reducción de dosis de acuerdo al riesgo aterotrombótico y de sangrado, así como minimizar el tiempo de terapia antitrombótica dual o triple ${ }^{23}$.

\section{Cardiopatía isquémica crónica}

En pacientes con SICA antiguo (> 1 año) con FA de reciente inicio se ha demostrado que la anticoagulación oral sin un agente antiplaquetario adicional es suficiente para el adecuado tratamiento de la mayoría de pacientes con FA y enfermedad arterial coronaria estable ${ }^{23}$.

Los AOD pueden ser una alternativa segura y eficaz a los AVK. En el caso de que se decida usar dabigatrán, debe considerarse la dosis más baja (110 mg 2 veces al día), además de adicionar dosis bajas de AAS (o clopidogrel en caso de alergia al AAS) en pacientes con alto riesgo aterotrombótico y bajo riesgo de hemorragia ${ }^{23}$.

\section{Cardioversión en pacientes bajo tratamiento con anticoagulantes orales directos}

Los pacientes con FA frecuentemente requieren de la cardioversión eléctrica o farmacológica para restaurar rápidamente el ritmo sinusal o para mejorar los síntomas. Sin embargo, esta se asocia a un riesgo incrementado de EVC perioperatorio o de ES. El riesgo de presentar eventos tromboembólicos es alto, del 5 al 7\% si la anticoagulación es inadecuada y del 0.7 al $0.8 \%$, con anticoagulación en rango terapéutico $^{36,37}$.

Las guías de la ESC recomiendan que a los pacientes con FA de más de $48 \mathrm{~h}$ de duración o FA con duración no determinada que se sometan a cardioversión se les debe iniciar anticoagulación oral al menos 3 semanas antes del procedimiento, o realizar un ecocardiograma transesofágico (TEE) para excluir la presencia de trombos en la aurícula izquierda. Posterior a la cardioversión se debe continuar la anticoagulación oral por 4 semanas más ${ }^{23}$.

Desde la aparición de los AOD, una gran cantidad de estudios han demostrado su beneficio en comparación con los AVK. Sin embargo, la información de la eficacia y la seguridad de los AOD en pacientes con FANV sometidos a cardioversión eléctrica o farmacológica es limitada. Se han realizado diferentes análisis post hoc de estudios clínicos a gran escala (RE-LY, ROCKET-AF, ARISTOTLE) para valorar desenlaces posteriores a la cardioversión ${ }^{36,37}$.

En el análisis post hoc del estudio RE-LY se comparó dabigatrán 110 y $150 \mathrm{mg}$ cada $12 \mathrm{~h}$ contra warfarina. Se realizaron un total de 1,983 cardioversiones en 1,270 pacientes. Se encontró que la frecuencia de EVC y de hemorragia mayor a los 30 días de la cardioversión con las 2 dosis de dabigatrán fue baja y comparable con warfarina con o sin TEE. Se concluyó que dabigatrán es una alternativa razonable en pacientes que requieran cardioversión. Los resultados se muestran en la tabla $6^{38}$.

El análisis post hoc del estudio ARISTOTLE comparó apixabán vs. warfarina. Se realizaron un total de 743 cardioversiones en 540 pacientes: 265 en pacientes tratados con apixabán y 275 con warfarina. Las características y los resultados se resumen en la tabla 7. En este estudio se observó que el EVC y el ES son poco frecuentes en los primeros 30 días de la cardioversión de la FA y la tasa es comparable entre apixabán y warfarina ${ }^{39}$.

En el análisis post hoc del estudio ROCKET-AF cuyo objetivo fue describir la incidencia, los predictores y los desenlaces asociados a cardioversión y ablación con catéter en pacientes tratados con rivaroxabán y warfarina, se incluyeron 321 pacientes que se sometieron a 460 procedimientos durante un seguimiento de 2.1 años. Un total de 143 fueron sometidos a cardioversión eléctrica (CVE), 142 a cardioversión farmacológica (CVF) y 79 a ablación con catéter. No se encontró diferencia significativa en los desenlaces a largo plazo (tiempo para el primer evento, riesgo de EVC o ES, muerte cardiovascular, muerte por todas las causas) antes y después de los procedimientos. El riesgo de 
Tabla 6 Eficacia de dabigatrán en comparación con warfarina en pacientes que se sometieron a cardioversión

\begin{tabular}{|c|c|c|c|c|c|c|}
\hline $\mathrm{n}=1,983$ & $\begin{array}{l}\text { \% con TEE } \\
\text { antes de la } \\
\text { cardioversión }\end{array}$ & $\begin{array}{l}\text { Evidencia de } \\
\text { trombo en Al }\end{array}$ & $\begin{array}{l}\% \\
\text { Mantenimiento } \\
\text { de la terapia } \\
\text { anticoagulante } \\
\geq 3 \text { semanas }\end{array}$ & $\begin{array}{l}\text { \% EVC y ES } \\
\text { en } 30 \text { días }\end{array}$ & $\begin{array}{l}\text { \% Hemorragia } \\
\text { mayor }\end{array}$ & $\begin{array}{l}\text { EVC y } \\
\text { hemorragia } \\
\text { en } 30 \text { días }\end{array}$ \\
\hline $\begin{array}{l}\text { Dabigatrán } 110 \mathrm{mg} \\
\quad(\mathrm{n}=647)\end{array}$ & 25 & 1.8 & $\begin{array}{l}76.4(\mathrm{p}<0.01 \\
\text { vs. warfarina) }\end{array}$ & $\begin{array}{l}0.8(p=0.71 \text { vs. } \\
\text { warfarina })\end{array}$ & $\begin{array}{l}1.7(p=0.06 \text { vs. } \\
\text { warfarina) }\end{array}$ & $\begin{array}{l}\text { Comparable } \\
\text { a warfarina } \\
\text { con y sin TEE }\end{array}$ \\
\hline $\begin{array}{l}\text { Dabigatrán } 150 \mathrm{mg} \\
\qquad(\mathrm{n}=672)\end{array}$ & 24.1 & 1.2 & $\begin{array}{l}79.2(p<0.01 \\
\text { vs. warfarina })\end{array}$ & $\begin{array}{l}0.3(p=0.40 \text { vs. } \\
\text { warfarina })\end{array}$ & $\begin{array}{l}0.6(p=0.99 \text { vs. } \\
\text { warfarina) }\end{array}$ & $\begin{array}{l}\text { Comparable } \\
\text { a warfarina } \\
\text { con y sin TEE }\end{array}$ \\
\hline Warfarina $(n=664)$ & 13.3 & 1.1 & 85.5 & 0.6 & 0.6 & - \\
\hline
\end{tabular}

Al: ataque isquémico; ES: embolismo sistémico; EVC: evento vascular cerebral; TEE: ecocardiograma transesofágico. Modificado de Nagarakanti et al. ${ }^{38}$.

\begin{tabular}{|c|c|c|}
\hline$n=540$ & $\begin{array}{l}\text { Apixabán } \\
(\mathrm{n}=265)\end{array}$ & $\begin{array}{l}\text { Warfarina } \\
(\mathrm{n}=275)\end{array}$ \\
\hline Trombo en Al & 0 & 0 \\
\hline $\begin{array}{l}\text { Tiempo para la primera } \\
\text { cardioversión (días) }\end{array}$ & $243 \pm 231$ & $252 \pm 248$ \\
\hline $\begin{array}{l}\text { Mínima duración con } \\
\text { anticoagulación para } \\
\text { cardioversión (días) }\end{array}$ & 1 & 4 \\
\hline $\begin{array}{l}\text { EVC o ES (30 días } \\
\text { poscardioversión) }\end{array}$ & 0 & 0 \\
\hline Infarto del miocardio & $0.3 \%$ & $0.2 \%$ \\
\hline Hemorragia mayor & $0.3 \%$ & $0.2 \%$ \\
\hline Muerte & $0.6 \%$ & $0.5 \%$ \\
\hline
\end{tabular}

Al: ataque isquémico; ES: embolismo sistémico; EVC: evento vascular cerebral.

Adaptado de Flaker et al. ${ }^{39}$.

hospitalización (HR: 2.01; IC 95\%: 1.51-2.68, p<0.0001) y la hemorragia clínicamente relevante mayor y menor (HR: 1.51; IC 95\%: 1.12-2.05, $\mathrm{p}<0.0072$ ) fueron mayores después de los procedimientos. La tasa de EVC y ES (1.88 vs. $1.86 \%)$ y muerte por todas las causas ( 1.88 vs. $3.73 \%$ ) fueron similares en el grupo de rivaroxabán y warfarina, respectivamente. En conclusión, salvo por el aumento en la hospitalización, no se encontró diferencia significativa en los desenlaces a largo plazo tras los procedimientos. En los otros desenlaces se demostró que todos los procedimientos fueron similares en pacientes tratados con rivaroxabán o warfarina ${ }^{40}$.

$\mathrm{El}$ explore the efficacy and safety of once-daily oral riVaroxaban for the prevention of caRdiovascular events in patients with non valvular aTrial fibrillation scheduled for cardioversion (X-VeRT) es el primer estudio prospectivo y aleatorizado con los AOD (rivaroxabán) en pacientes con FA expuestos a cardioversión. Un total de 1,504 pacientes fueron distribuidos al azar en una proporción 2:1 rivaroxabán $(n=1,002)$ y AVK $(n=502)$. Se realizó cardioversión electiva temprana (dentro de uno a 5 días) o tardía ( 3 a 8 semanas) en ambos grupos. Se investigaron los desenlaces de EVC, AIT, ES, IAM, muerte CV y hemorragia mayor. Los resultados se muestran en la tabla 8 . En conclusión, se obtuvo que el rivaroxabán es una alternativa eficaz y segura a los AVK, además de que puede permitir que la cardioversión sea más temprana ${ }^{41}$.

La evidencia actual, aunque limitada aún, proporciona información valiosa para el empleo de los AOD en los pacientes candidatos a cardioversión de la FA, con resultados de eficacia y seguridad comparables a los $\mathrm{AVK}^{36}$.

\section{Casos especiales}

\section{Pacientes con enfermedad renal crónica}

La ERC constituye un factor de riesgo para presentar hemorragias y eventos tromboembólicos en pacientes con FA, particularmente con depuración de creatinina $<60 \mathrm{ml} / \mathrm{min}^{23}$.

Las nuevas guías recomiendan el uso de los AOD en pacientes con enfermedad renal leve o moderada haciendo los ajustes de dosis recomendados para cada fármaco (tabla 9). El apixabán se puede utilizar con DCr entre 15 y $29 \mathrm{ml} / \mathrm{min}$, con ajuste de dosis de $2.5 \mathrm{mg} 2$ veces al día. Con una $\mathrm{DCr}$ entre 15 y $49 \mathrm{ml} / \mathrm{min}$ se recomienda la reducción de dosis de rivaroxabán a $15 \mathrm{mg}$ al día. El dabigatrán fue aprobado por la FDA a dosis de $75 \mathrm{mg}$ en pacientes con $\mathrm{DCr}$ de 15 a $30 \mathrm{ml} / \mathrm{min}$ mediante modelos farmacológicos pero no en estudios clínicos. En el caso de una $\mathrm{DCr}<15 \mathrm{ml} / \mathrm{min}$ o pacientes en hemodiálisis no se recomienda el uso de AOD: en ellos la warfarina se considera como el anticoagulante de elección ${ }^{2,21}$.

Es importante también tener en cuenta las especificaciones individuales de los AOD para poder elegir el que brinde mayor beneficio al paciente (tabla 10) $)^{23}$. También se sugiere una monitorización cada 6 meses en pacientes con enfermedad renal leve y cada 3 meses cuando es moderada ${ }^{21}$.

\section{Nuevos anticoagulantes en prevención del evento vascular cerebral en el adulto mayor con fibrilación auricular no valvular}

A pesar de que la prevalencia de la FA y el riesgo de EVC aumentan con la edad, se ha visto que los adultos mayores 
Tabla 8 Resultados del estudio X-VeRT

\begin{tabular}{|c|c|c|c|c|c|c|c|c|}
\hline$n=1504$ & $\begin{array}{l}\text { \% ocurrencia de } \\
\text { desenlaces con } \\
\text { cardioversión } \\
\text { temprana }\end{array}$ & $\begin{array}{l}\text { \% ocurrencia de } \\
\text { desenlaces con } \\
\text { cardioversión } \\
\text { tardía }\end{array}$ & $\%$ EVC & $\%$ AIT & $\%$ ES & $\%$ IAM & $\begin{array}{l}\% \text { muerte } \\
\mathrm{CV}\end{array}$ & $\begin{array}{l}\text { Hemorragia } \\
\text { mayor }\end{array}$ \\
\hline $\begin{array}{l}\text { Rivaroxabán } \\
\quad(n=978)\end{array}$ & 0.71 & 0.24 & 0.2 & 0 & 0 & 0.1 & 0.41 & 0.61 \\
\hline Warfarina $(n=492)$ & 1.08 & 0.93 & 0.41 & 0 & 0.2 & 0.2 & 0.41 & 0.80 \\
\hline
\end{tabular}

AIT: ataque isquémico transitorio; CV: cardiovascular; ES: embolismo sistémico; EVC: evento vascular cerebral; IAM: infarto agudo de miocardio.

Modificado de Cappato et al. ${ }^{41}$.

Tabla 9 Recomendaciones de ajuste de dosis en pacientes con enfermedad renal en tratamiento con nuevos anticoagulantes orales

\begin{tabular}{llll}
\hline Función renal & Apixabán & Dabigatrán & Rivaroxabán \\
\hline Normal/ERC leve & 5 o $2.5 \mathrm{mg} \mathrm{c} / 12 \mathrm{~h}$ & $150 \mathrm{mg} \mathrm{c} / 12 \mathrm{~h}(\mathrm{DCr}>30 \mathrm{ml} / \mathrm{min})$ & $20 \mathrm{mg} \mathrm{c} / 24 \mathrm{~h}(\mathrm{DCr}>50 \mathrm{ml} / \mathrm{min})$ \\
ERC moderada & 5 o $2.5 \mathrm{mg} \mathrm{c} / 12 \mathrm{~h}$ & $150 \mathrm{mg} \mathrm{c} / 12 \mathrm{~h}(\mathrm{DCr}>30 \mathrm{ml} / \mathrm{min})$ & $15 \mathrm{mg} \mathrm{c} / 24 \mathrm{~h}(\mathrm{DCr} 30-50 \mathrm{ml} / \mathrm{min})$ \\
ERC severa & No se recomienda & $75 \mathrm{mg} \mathrm{c} / 12 \mathrm{~h}(\mathrm{DCr} 15-30 \mathrm{ml} / \mathrm{min})$ & $15 \mathrm{mg} \mathrm{c} / 24 \mathrm{~h}(\mathrm{DCr} 15-30 \mathrm{ml} / \mathrm{min})$ \\
ERC sin diálisis & No se recomienda & No se recomienda & No se recomienda \\
ERC con diálisis & No se recomienda & No se recomienda & No se recomienda \\
\hline
\end{tabular}

DCr: depuración de creatinina; ERC: enfermedad renal crónica.

Modificado de January et al. ${ }^{2}$.

Tabla 10 Absorción y eliminación renal de los anticoagulantes orales

\begin{tabular}{llll}
\hline AOD & $\begin{array}{l}\text { \% dosis } \\
\text { absorbida } \\
\text { excretada } \\
\text { en el riñón }\end{array}$ & $\begin{array}{l}\text { \% dosis } \\
\text { adminis- } \\
\text { trada } \\
\text { excretada } \\
\text { por el riñón }\end{array}$ & $\begin{array}{l}\text { Aprobada } \\
\text { para esta } \\
\mathrm{DCr} \\
(\mathrm{ml} / \mathrm{min})\end{array}$ \\
\hline $\begin{array}{l}\text { Dabigatrán } \\
\text { Rivaroxabán }\end{array}$ & 80 & 4 & $>30$ \\
Apixabán & 27 & 33 & $>15$ \\
\hline
\end{tabular}

AOD: anticoagulantes orales directos; $\mathrm{DCr}$ : depuración de creatinina.

Modificado de Heidbuchel et al. ${ }^{23}$.

se encuentran subtratados con $\mathrm{AVK}^{32}$. Múltiples estudios han encontrado que los pacientes geriátricos reciben menos terapia anticoagulante que los pacientes de menor edad ${ }^{42}$.

En un estudio retrospectivo en el cual se revisaron las admisiones hospitalarias de pacientes con FA y EVC isquémico se observó que el $75 \%$ de los pacientes menores de 75 años recibieron anticoagulación, comparado con el 33\% de los mayores de 85 años. De igual manera, otro estudio prospectivo encontró que la tasa de disminución de anticoagulación aumenta con la edad, siendo del $75 \%$ en pacientes de 65 a 69 años, del $59 \%$ con 70 a 79 años, del $45 \%$ con 80 a 89 años y del $24 \%$ en mayores de 90 años tratados con warfarina $^{42}$. Esto se debe a las limitaciones de los AVK, la preocupación de mayor riesgo de hemorragia y caídas, así como a las interacciones farmacológicas y alimentarias ${ }^{42,43}$.

Los AOD han demostrado tener eficacia mayor o similar a la warfarina en la prevención de EVC; así mismo, se asocian a riesgo menor o equivalente en la disminución de hemorragia. Los 4 estudios más recientes de AOD en FANV en adultos mayores de 75 años de edad incluyeron 21,062 pacientes $y$ ofrecen una sustancial evidencia de su eficacia en este grupo de edad. En la tabla 11 se muestran los resultados ${ }^{44}$.

En los adultos mayores se deben tomar consideraciones especiales durante el tratamiento con los AOD, ya que debido a su edad presentan una disminución en la función renal, menos peso corporal y se encuentran polimedicados, lo que ocasiona un mayor número de interacciones farmacológicas que los pacientes más jóvenes ${ }^{42}$.

En pacientes $\geq 75$ años la concentración plasmática del dabigatrán aumenta hasta 1.3 veces, en $\geq 65$ años se incrementa 1.5 veces más para rivaroxabán y en > 65 años es 1.3 veces mayor para apixabán. Debido a estas variaciones, en este grupo de pacientes se requieren ajustes de las dosis de AOD. Pacientes en tratamiento con apixabán deben ser tratados con una dosis más baja de $2.5 \mathrm{mg} 2$ veces al día si cumplen con al menos 2 características: $\geq 80$ años, peso $\leq 60 \mathrm{~kg}$ y creatinina sérica $\geq 1.5 \mathrm{mg} / \mathrm{dl}$. Para dabigatrán se sugieren un ajuste de dosis de $75 \mathrm{mg}$ en mayores de 80 años. En cuanto a rivaroxabán, no se cuenta con estudios donde se indique el ajuste de dosis en adultos mayores $^{42}$.

Para mantener una mayor adherencia y seguridad en el uso de los AOD es indispensable tener en cuenta las recomendaciones para su prescripción y que el paciente reconozca que el efecto anticoagulante declina rápidamente en 12 a $24 \mathrm{~h}$ al dejar de tomarlos. La educación al paciente y su familia, tener una hoja de instrucciones, tablas de seguridad y sesiones grupales pueden mejorar la adhesión, la seguridad y la eficacia de los AOD en los adultos mayores con FANV. 
Tabla 11 Comparación de AOD en adultos mayores de 75 años

\begin{tabular}{|c|c|c|c|c|c|}
\hline$A O D$ & Estudio & Dosificación & > 75 años (n) & $\begin{array}{l}\text { HR riesgo } \\
\text { EVC }\end{array}$ & $\begin{array}{l}\text { HR hemorragia } \\
\text { mayor }\end{array}$ \\
\hline \multirow[t]{2}{*}{ Dabigatrán } & RE-LY & $110 \mathrm{mg} \mathrm{c} / 12 \mathrm{~h}$ & 7,250 & 0.88 & 1.01 \\
\hline & & $150 \mathrm{mg} \mathrm{c} / 12 \mathrm{~h}$ & 7,258 & 0.67 & 1.18 \\
\hline Rivaroxabán & ROCKET-AF & $\begin{array}{l}-20 \mathrm{mg} / \mathrm{d} \\
-15 \mathrm{mg} / \mathrm{d}(\mathrm{DCr} 30-49 \mathrm{ml} / \mathrm{min})\end{array}$ & 6,229 & 0.88 & 1.04 \\
\hline \multirow{2}{*}{ Apixabán } & ARISTOTLE & $5 \mathrm{mg} \mathrm{c} / 12 \mathrm{~h}$ & 5,678 & 0.79 & 0.69 \\
\hline & AVERROES & $\begin{array}{l}-5 \mathrm{mg} \mathrm{c} / 12 \mathrm{~h} \\
-2.5 \mathrm{mg} \mathrm{c} / 12 \text { con } 2 \text { o más: (CrS } \\
\geq 133 \mathrm{ml} / \mathrm{min}, \geq 80 \text { años, } \leq 60 \mathrm{~kg} \text { ) vs. AAS }\end{array}$ & 1,897 & 0.46 & 1.13 \\
\hline
\end{tabular}

AOD: anticoagulantes orales directos; DCr: depuración de creatinina; EVC: evento vascular cerebral; HR: riesgo de hospitalización. Modificado de $\mathrm{Ng}$ et al. ${ }^{44}$.

\section{Manejo perioperatorio de los nuevos anticoagulantes orales}

Para poder entender la forma de suspender y reiniciar los AOD de manera previa a intervenciones quirúrgicas o procedimientos intervencionistas es fundamental tomar en cuenta diferentes factores determinantes de la conducta respecto a las modificaciones del tratamiento antitrombótico. Es necesario comprender adecuadamente las características farmacodinámicas y farmacocinéticas del anticoagulante empleado, tener en cuenta las comorbilidades de los pacientes (en especial la función renal) y los factores relacionados a la cirugía (si es electiva o de urgencia), así como el riesgo de sangrado del procedimiento ${ }^{45}$.

Según las guías de la EHRA, se recomienda clasificar los procedimientos quirúrgicos en 3 categorías: intervenciones que no requieren descontinuación del anticoagulante y procedimientos con riesgo bajo y alto de hemorragia ${ }^{23}$.

Las intervenciones que no requieren descontinuación, como las dentales, las oftalmológicas para glaucoma 0 cataratas, la endoscopia y las cirugías superficiales, como incisión de absceso o escisiones dermatológicas pequeñas, se pueden realizar después de 12 a $24 \mathrm{~h}$ de la última toma de AOD (18 a $24 \mathrm{~h}$ de preferencia) y reiniciar el tratamiento $6 \mathrm{~h}$ tras a la intervención ${ }^{23}$.

En pacientes con adecuada función renal, en los procedimientos con bajo riesgo de sangrado como la endoscopia sin biopsia, la biopsia prostática o vesical, la angiografía y la implantación de marcapasos o desfibrilador se recomienda suspender el AOD $24 \mathrm{~h}$ antes de la cirugía electiva. En el caso de alto riesgo de sangrado, como en las cirugías torácica, abdominal y ortopédica mayores, la RTUP, la biopsia hepática o renal, la anestesia espinal o epidural y la punción lumbar, se recomienda tomar la última dosis de AOD $48 \mathrm{~h}$ antes. En cuanto a los pacientes con enfermedad renal, se sugieren diferentes indicaciones para cada AOD (fig. 2$)^{23}$.

Procedimientos con hemostasia completa e inmediata, anestesia espinal o epidural atraumática o punción lumbar limpia, el reinicio de AOD se realiza a las 6 a $8 \mathrm{~h}$ posteriores. Sin embargo, si el riesgo de hemorragia es mayor al de trombosis, se debe reiniciar el tratamiento después de 48 a $72 \mathrm{~h}$ del procedimiento. En caso de que el paciente tenga que estar inmovilizado y, por consiguiente, aumente su riesgo de tromboembolismo, se requiere tromboprofilaxis con HBPM a las 6 a $8 \mathrm{~h}$ posquirúrgicas y reiniciar el AOD hasta las 48 a $72 \mathrm{~h}$ después de la cirugía $\mathrm{a}^{23,45}$.

Debido a que no se cuenta con antídotos específicos para los AOD, el manejo perioperatorio de cirugías de emergencia o por trauma es de especial preocupación. Si es posible, se sugiere posponer la cirugía $12 \mathrm{~h}$ (idealmente $24 \mathrm{~h}$ ) después de la última dosis del AOD. Si la ingesta fue $2 \mathrm{~h}$ antes del ingreso, se puede utilizar carbón activado, hemodiálisis en pacientes con enfermedad renal en tratamiento con dabigatrán y, en caso de hemorragia severa, como HIC, utilizar CCP o plasma fresco congelado ${ }^{46,47}$.

\section{Costo-beneficio de la anticoagulación en la fibrilación auricular}

Las hospitalizaciones frecuentes, las alteraciones hemodinámicas y los eventos tromboembólicos relacionados con la FA causan una gran morbimortalidad y costos muy elevados para su atención médica. Los pacientes con FA tienen un riesgo 5 veces mayor para desarrollar embolias cerebrales, y el cual aumenta con la edad, además de incrementarse 3 veces más el riesgo de IC y 2 veces el riesgo de demencia y mortalidad.

Las hospitalizaciones por FA son el diagnóstico primario en más de 467,000 personas anualmente en Estados Unidos y contribuyen a más de 99,000 muertes por año. Estos pacientes tienen 20 veces más probabilidad de ser hospitalizados y 3 veces más de rehospitalización, generando un costo aproximado de $\$ 8,700$ dólares/persona por año (estimado entre 2004 a 2006). El costo calculado de todos los pacientes tratados por FA es de $\$ 26$ billones de dólares anuales en los servicios de salud de Estados Unidos. Considerando que la FA afecta entre 2.7 y 6.1 millones de adultos americanos, se espera que el número se duplique en los siguientes 25 años ${ }^{2}$.

Las diferencias en los costos médicos de los AOD en comparación con la warfarina dependen del AOD específico utilizado y del riesgo de EVC. En este análisis, apixabán se asoció con costos médicos reducidos consistentemente respecto a la warfarina en pacientes con FANV con riesgo moderado o alto. Los principales indicadores de la reducción de costos médicos asociados con el uso de apixabán en relación con la warfarina fueron la reducción de las tasas de embolia cerebral, siendo 14 y $32 \%$ menor, y la hemorragia 


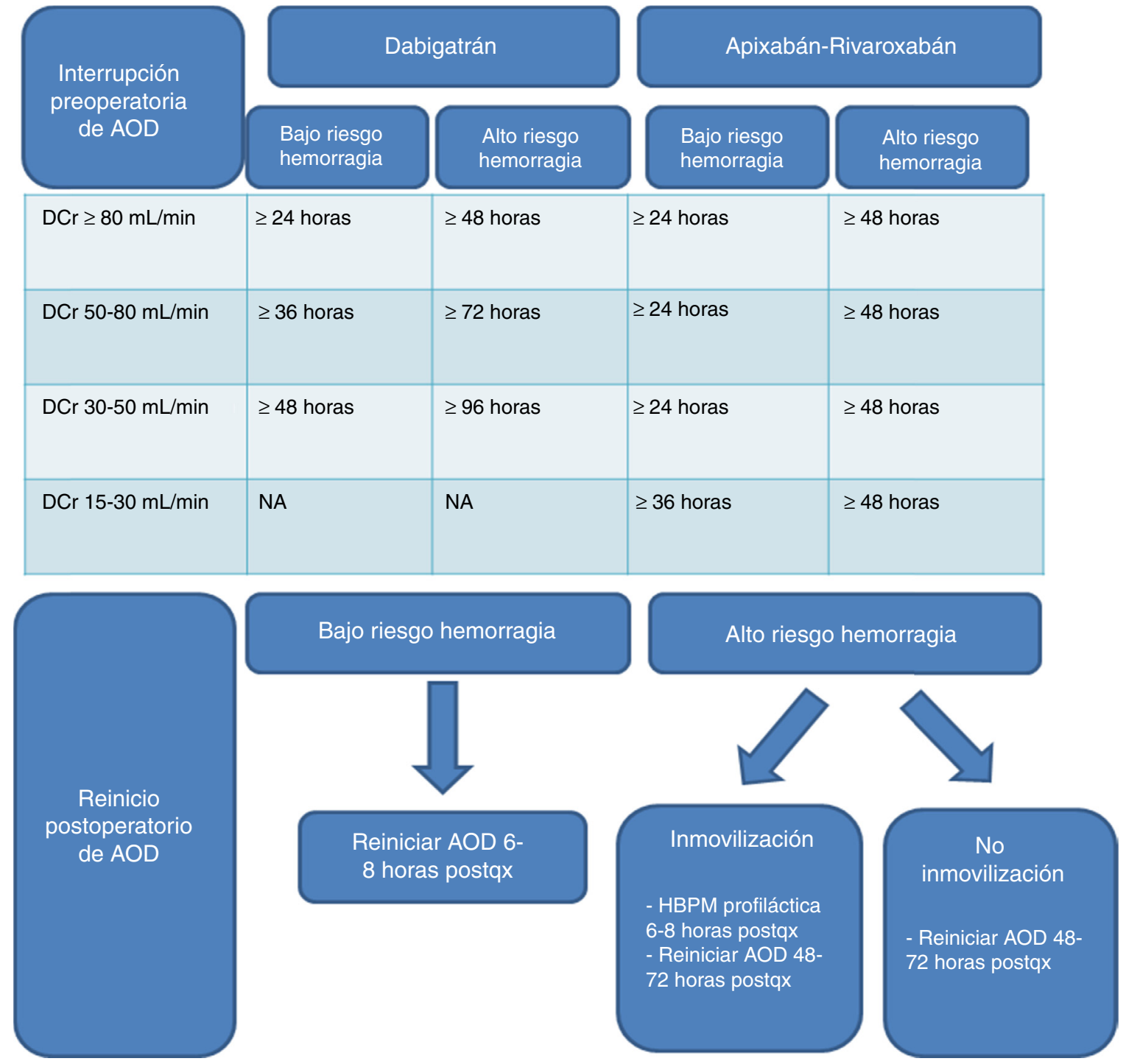

Figura 2 Indicaciones de anticoagulantes orales directos en pacientes con enfermedad renal.AOD: anticoagulantes orales directos; DCr: depuración de creatinina; HBPM: heparina de bajo peso molecular; NA: no aplica.

Modificado de Lai et al. ${ }^{46}$, Faraoni et al. ${ }^{45}$, Heidbuchel et al. ${ }^{23}$.

mayor, que disminuyó 20 y $25 \%$ para los pacientes con FANV $\mathrm{CHADS}_{2}=2$ y $\mathrm{CHADS}_{2} \geq 3$, respectivamente ${ }^{48}$.

El uso de dabigatrán o rivaroxabán en lugar de warfarina se asoció con un menor riesgo de embolia cerebral, pero los riesgos no fueron consistentemente más bajos para otros eventos clínicos y, por lo tanto, su diferencia de costos fue menor. Este análisis se basa en datos de ensayos clínicos, y la aplicación de los resultados a la práctica clínica habitual requiere una evaluación adicional. Debido a que la incidencia de EVC y de hemorragia puede ser mayor en la práctica clínica habitual, la diferencia de costos puede haber sido subestimada. Además, los eventos de hemorragia y sus tasas relativas fueron reportados originalmente como cocientes de riesgo, siendo que el análisis mide el riesgo relativo de «tiempo hasta el primer evento». El enfoque utilizado en este análisis no consideró ningún impacto de oportunidad y, por lo tanto, puede haber subestimado la tasa de hemorragia mayor. Para los pacientes con FANV con riesgo moderado para presentar un EVC $\left(\mathrm{CHADS}_{2}=2\right)$ las diferencias en los costos médicos de eventos clínicos frente a la warfarina fueron: $-298,-143$ y +117 dólares por paciente y año para apixabán, dabigatrán $(150 \mathrm{mg})$ y rivaroxabán, respectivamente (los números negativos indican reducción de costos). Para los pacientes con alto riesgo de EVC $\left(\mathrm{CHADS}_{2} \geq 3\right)$ la diferencia en costo de gastos médicos vs. warfarina fue de $-697,+2$ y -100 dólares para apixabán, dabigatrán (150 mg) y rivaroxabán, respectivamente ${ }^{48}$.

En Europa existen artículos realizados en poblaciones con características clínicas similares y analizando el uso de los AOD en pacientes con FA comparados con warfarina. En el estudio realizado por González-Juanatey et al. ${ }^{49}$ se realizó una adaptación de un modelo de Markov que simula la historia natural de la enfermedad para una cohorte de 10,000 pacientes con FANV a lo largo de su vida. Se comparó el dabigatrán con warfarina en un primer escenario y el patrón de prescripción habitual en la práctica clínica española (60\% con AVK, $30 \%$ con AAS y $10 \%$ sin tratamiento) en el segundo. Se realizaron análisis de sensibilidad y 


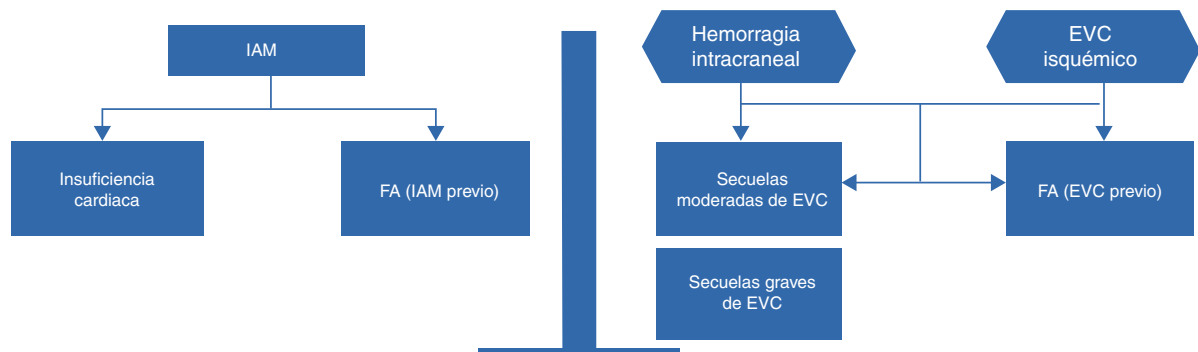

Muerte

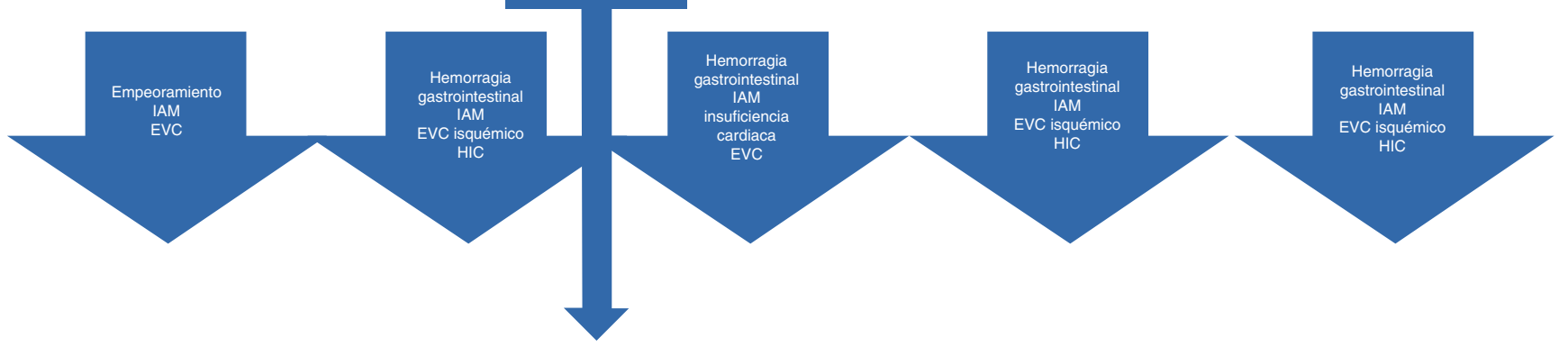

Muerte

Figura 3 Modelo estructural de los eventos que ocurren en pacientes con fibrilación auricular que aumentan la morbimortalidad, generando costos adicionales al tratamiento individualizado.

EVC: evento vascular cerebral; FA: fibrilación auricular; HIC: hemorragia intracraneal; IAM: infarto agudo de miocardio. Modificado de Wisløff et al. ${ }^{50}$.

probabilidad. En ambos escenarios, dabigatrán disminuyó el número de eventos presentados, reflejándose una mejoría en cantidad y calidad de vida. El incremento en la razón costo-efectividad de dabigatrán fue de 17,581 y de 14,118 euros/año de vida ajustado por calidad ganada comparado con warfarina y respecto al patrón de prescripción habitual, respectivamente. Incorporando los costos sociales al análisis, dabigatrán es una estrategia dominante (más efectiva y de menor costo). Desde la perspectiva del Sistema Nacional de Salud, dabigatrán resulta una estrategia eficiente para la prevención de EVC en pacientes con FANV en comparación con warfarina y con el patrón de prescripción habitual, ya que en ambas comparaciones realizadas los valores de incremento de la razón costo-efectividad estuvieron por debajo del umbral de 30,000 euros/año de vida ajustado por calidad.

Asimismo, se realizó un estudio con el objetivo de evaluar la relación costo-eficacia de warfarina, dabigatrán, rivaroxabán y apixabán en un entorno de salud pública en Noruega, creando un modelo probabilístico de Markov analítico de decisión para simular la vida de los pacientes con FA. Se realizaron análisis estadísticos en diferentes escenarios, incluyendo el cambio de la edad para dabigatrán de 80 a 75 años. Siguiendo las recomendaciones de las guías de la Sociedad Europea de Cardiología, dabigatrán $(150 \mathrm{mg}$ 2 veces al día hasta los 80 años de edad y $110 \mathrm{mg} 2$ veces al día a partir de los 80 años), parece ser la alternativa más rentable para los pacientes con FA de alto riesgo. Para los pacientes con riesgo intermedio, apixabán $(5 \mathrm{mg}, 2$ veces al día) parece ser un poco más eficaz que el dabigatrán, pero dabigatrán sigue siendo la alternativa más rentable. En el análisis del escenario donde se redujo dabigatrán de $150 \mathrm{mg}$ 2 veces al día a $110 \mathrm{mg} 2$ veces al día a partir de los 75 años de edad en lugar de a los 80 años, apixabán $(5 \mathrm{mg}, 2$ veces al día) resultó ser la alternativa más costo-efectiva para ambos grupos de riesgo ${ }^{50}$.

Ahora hay que recordar que existen indicaciones específicas para el uso de warfarina e indicaciones específicas para los AOD en la prevención de eventos cardioembólicos en pacientes con FANV, por lo que cada caso debe ser individualizado. Los AOD incrementan el armamentario terapéutico y son una estrategia que mejorará en gran medida el futuro de la anticoagulación. Sin embargo, con la evidencia actual se deben considerar con el paciente los beneficios, los riesgos y la conveniencia del costo-efectividad aplicados a la práctica clínica diaria considerando los aspectos trascendentes a la hora de la vida real y no deben ser recomendados todos los AO como una panacea o como el tratamiento de primera línea para todos los pacientes (fig. 3$)^{50}$.

Muchos de estos pacientes, particularmente los que tienen un alto riesgo tromboembólico y de hemorragia con pobre control del INR o que no aceptan el uso de terapia con warfarina (principalmente por las restricciones alimentarias y farmacológicas, así como la necesidad de estudios de laboratorio frecuentes), son los que probablemente se beneficiarán más con los AOD. Para los siguientes años, a estos grupos de pacientes se les ofrecerá alguno de estos como de primera línea, se ampliarán las indicaciones, se conocerá 
más sobre la seguridad de los mismos y el costo-beneficio seguirá mejorando incluso entre cada uno de ellos en particular y probablemente la warfarina vaya perdiendo lugar como el anticoagulante más comúnmente utilizado para los pacientes con FANV ${ }^{51}$.

\section{Oclusores de orejuela izquierda en pacientes con contraindicación o intolerancia a los anticoagulantes orales}

Para los pacientes que tienen contraindicación o no toleran los $\mathrm{AO}$ se han desarrollado nuevas técnicas para reducir el riesgo de un $\mathrm{EVC}^{52}$. Como ya es bien sabido, la mayoría de los trombos en pacientes con FANV se forman en la orejuela izquierda, y por esta razón los oclusores de orejuela izquierda son una alternativa recomendada para reducir el riesgo de embolismos en este grupo de pacientes ${ }^{53}$. De las técnicas disponibles (quirúrgica y transcatéter), se ha estudiado más ampliamente la técnica transcatéter, la cual ha logrado no inferioridad vs warfarina y, a la vez, ser una alternativa segura y efectiva ${ }^{54}$.

\section{Financiamiento}

Se recibió patrocinio del laboratorio farmacéutico Pfizer para llevar a cabo este artículo.

\section{Conflicto de intereses}

Los autores declaran no tener ningún conflicto de intereses.

\section{Anexo 1.}

Tabla A1 Escala $\mathrm{CHADS}_{2}$

\begin{tabular}{lll}
\hline Letra & Característica clínica & Puntaje \\
\hline $\mathrm{C}$ & $\begin{array}{l}\text { CHF }=\text { insuficiencia } \\
\text { cardiaca congestiva }\end{array}$ & 1 \\
$\mathrm{H}$ & Hipertensión & 1 \\
$\mathrm{~A}$ & Age $=$ edad $\geq 75$ años & 1 \\
$\mathrm{D}$ & $\underline{\text { Diabetes mellitus }}$ & 1 \\
$\mathrm{~S}_{2}$ & Stroke $=$ EVC/AIT/TE & 2 \\
Puntaje máximo & & 6
\end{tabular}

AIT: ataque isquémico transitorio; EVC: evento cerebral vascular; TE: tromboembolia 0 puntos: bajo riesgo; 1 punto: riesgo intermedio; $\geq 2$ puntos: riesgo alto Incidencia anual Puntos ajustada de EVC

\begin{tabular}{ll} 
Puntos & $\begin{array}{l}\text { Incidencia } \\
(\%)\end{array}$ \\
0 & 1.9 \\
1 & 2.8 \\
2 & 4.0 \\
3 & 5.9 \\
4 & 8.5 \\
5 & 12.5 \\
6 & 18.2 \\
\hline
\end{tabular}

Anexo 2.

Tabla A2 Escala $\mathrm{CHA}_{2} \mathrm{DS}_{2}$-Vasc

\begin{tabular}{lll}
\hline Letra & Característica clínica & Puntaje \\
\hline C & Cardiaco $=$ ICC/ & 1 \\
& disfunción ventricular & \\
& izquierda \\
H & Hipertensión & 1 \\
$\mathrm{~A}$ & Age $=$ edad $\geq 75$ años & 1 \\
D & Diabetes mellitus & 1 \\
$S_{2}$ & Stroke $=$ EVC/AIT/TE & 2 \\
V & Vascular = enfermedad & 1 \\
& vascular & \\
A & Age = edad 65 a 74 años & 1 \\
Sc & Sex category = sexo & 1
\end{tabular}

Puntaje máximo 9

AIT: ataque isquémico transitorio; EVC: evento vascular cerebral; TE: tromboembolia, enfermedad vascular $=$ infarto previo del miocardio, enfermedad arterial periférica, aterosclerosis aórtica

0 puntos: bajo riesgo; 1 punto: riesgo intermedio;

$\geq 2$ puntos: riesgo alto

Incidencia anual Puntos Incidencia ajustada de EVC 


\section{Anexo 3.}

Tabla A3 Tarjeta de control de tratamiento con anticoagulantes orales directos

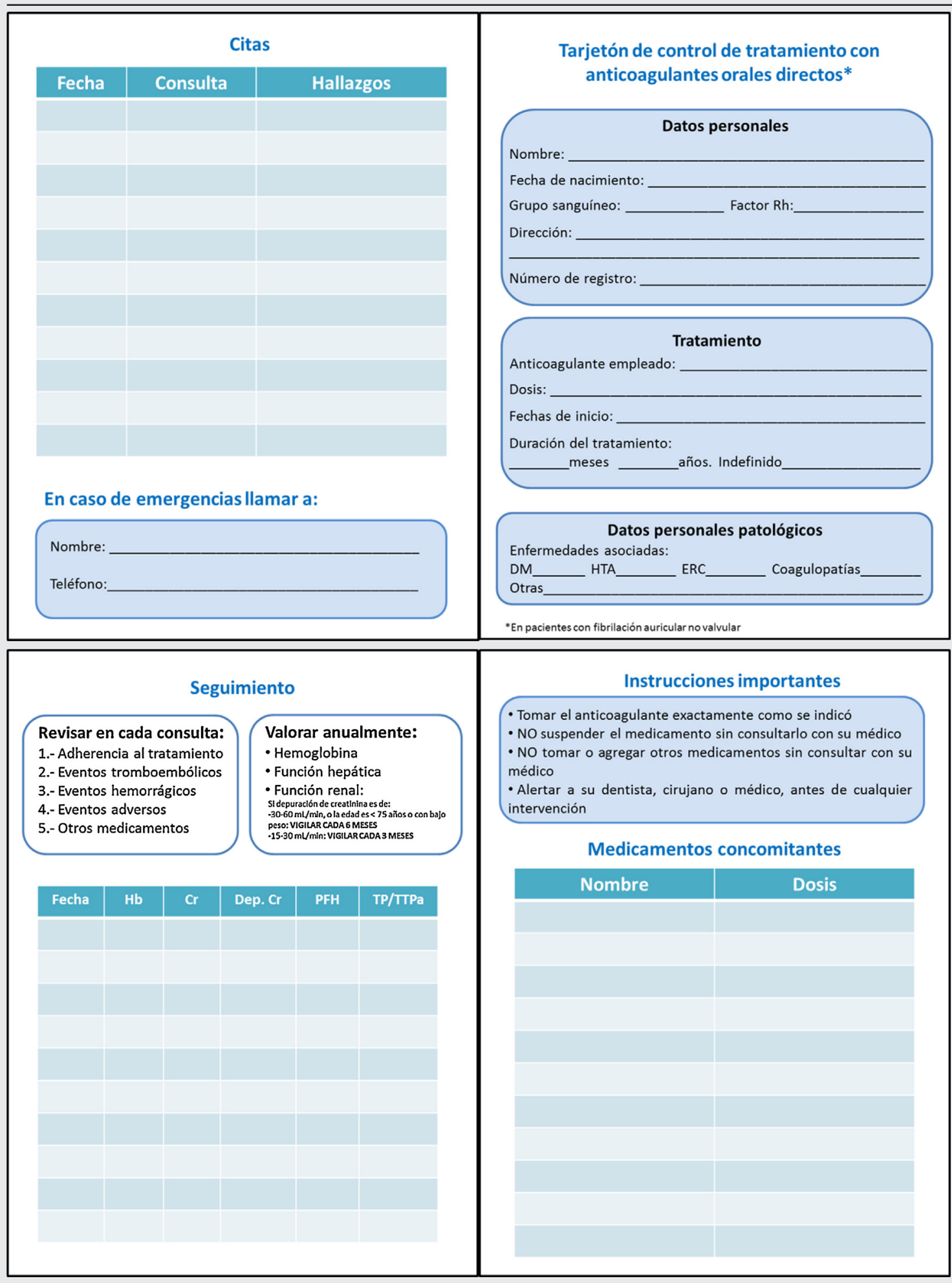

Adaptado de Heidbuchel et al. ${ }^{23}$. Tarjeta de control de anticoagulantes orales directos, del Instituto Nacional de Cardiología Ignacio Chávez, cortesía del Dr. Izaguirre. 


\section{Anexo 4.}

\section{Tabla A4 Escala HAS-BLED}

\begin{tabular}{|c|c|c|}
\hline Letra & Característica clínica & Puntaje \\
\hline $\mathrm{H}$ & $\begin{array}{l}\text { Hipertensión TAS } \\
\geq 160 \mathrm{mmHg}\end{array}$ & 1 \\
\hline A & $\begin{array}{l}\text { Anormal = disfunción } \\
\text { renal o hepática } \\
\text { (un punto cada uno) }\end{array}$ & $1 \circ 2$ \\
\hline$S$ & $\underline{\text { Stroke }}=$ historia de EVC & 1 \\
\hline B & $\begin{array}{l}\text { Bleeding = hemorragia } \\
\text { o anemia }\end{array}$ & 1 \\
\hline L & Lábil = INR inestable & 2 \\
\hline$E$ & $\begin{array}{l}\text { Elderly = pacientes } \\
\text { geriátricos (edad } \\
\geq 65 \text { años) }\end{array}$ & 1 \\
\hline D & $\begin{array}{l}\text { Drugs = alcohol o } \\
\text { medicamentos (un punto } \\
\text { cada uno) }\end{array}$ & $1 \circ 2$ \\
\hline Puntaje máximo & & 9 \\
\hline \multicolumn{3}{|c|}{$\begin{array}{l}\text { Disfunción renal: diálisis, trasplante renal o creati } \\
\text { sérica } \geq 2.6 \mathrm{mg} / \mathrm{dl} \text {; disfunción hepática: hepatopat } \\
\text { crónica (cirrosis); bilirrubinas } \geq 2 \text { veces normal; AS } \\
\text { FA } \geq 3 \text { veces normal; EVC: evento vascular cerebra } \\
\text { fármacos (antiplaquetarios, analgésicos, etc.). } \\
0 \text { puntos: bajo riesgo; } 1 \text { punto: riesgo intermedio; } \\
\geq 2 \text { puntos: riesgo alto }\end{array}$} \\
\hline $\begin{array}{l}\text { Incidencia anual } \\
\text { ajustada de }\end{array}$ & Puntos & $\begin{array}{l}\text { Incidencia } \\
(\%)\end{array}$ \\
\hline \multirow[t]{7}{*}{ hemorragia } & 0 & 1.13 \\
\hline & 1 & 1.02 \\
\hline & 2 & 1.88 \\
\hline & 3 & 3.74 \\
\hline & 4 & 8.7 \\
\hline & 5 & 12.5 \\
\hline & Cualquier punto & 1.56 \\
\hline
\end{tabular}

\section{Bibliografía}

1. Camm AJ, Kirchhof P, Lip GYH, et al., Guidelines Management of Atrial Fibrillation of the European Society of Cardiology. Guidelines for the management of atrial fibrillation. Eur Heart J. 2010;31:2369-429.

2. January CT, Wann LS, Alpert JS, et al. 2014 AHA/ACC/HRS guideline for the management of patients with atrial fibrillation: a report of the American College of Cardiology/American Heart Association Task Force on Practice Guidelines and the Heart Rhythm Society. J Am Coll Cardiol. 2014;64:e1-76.

3. Lara-Vaca S, Cordero-Cabra A, Martínez-Flores E, et al. Registro Mexicano de Fibrilación Auricular. Gaceta Méd México. 2014;150 Suppl 1:48-59.

4. Lega JC, Mismetti P, Cucherat M, et al. Impact of double-blind vs. open study design on the observed treatment effects of new oral anticoagulants in atrial fibrillation: A meta-analysis. J Thromb Haemost. 2013;11:1240-50.

5. Mateo J. Nuevos anticoagulantes orales y su papel en la práctica clínica. Rev Esp Cardiol Supl. 2013;13:33-41.
6. Ruff CT, Giugliano RP, Braunwald E, et al. Comparison of the efficacy and safety of new oral anticoagulants with warfarin in patients with atrial fibrillation: A meta-analysis of randomised trials. Lancet. 2014;383:955-62.

7. Pokorney SD, Sherwood MW, Becker RC. Clinical strategies for selecting oral anticoagulants in patients with atrial fibrillation. J Thromb Thrombolysis. 2013;36:163.

8. Gómez-Outes A, Terleira-Fernández Al, Calvo-Rojas G, et al. Dabigatran, rivaroxaban, or apixaban versus warfarin in patients with nonvalvular atrial fibrillation: A systematic review and meta-analysis of subgroups. Thrombosis. 2013;2013:640-723.

9. Granger CB, Alexander JH, McMurray JJ, et al. Apixaban versus warfarin in patients with atrial fibrillation. N Engl J Med. 2011;365:981-92.

10. Ageno W, Gallus AS, Wittkowsky A, et al. Oral anticoagulant therapy: Antithrombotic Therapy and Prevention of Thrombosis, 9th ed: American College of Chest Physicians Evidence-Based Clinical Practice Guidelines. Chest. 2012;141 2 Suppl, e44S-88S.

11. Alegret JM, Viñolas $X$, Arias MA, et al. New oral anticoagulants vs vitamin K antagonists: Benefits for health-related quality of life in patients with atrial fibrillation. Int J Med Sci. 2014;11:680-4.

12. Schirmer SH, Baumhäkel M, Neuberger HR, et al. Novel anticoagulants for stroke prevention in atrial fibrillation: Current clinical evidence and future developments. J Am Coll Cardiol. 2010;56:2067-76.

13. Harenberg J, Weiss C. Clinical trials with new oral anticoagulants. Additive value of indirect comparisons also named network meta-analyses. Hamostaseologie. 2013;33:62-70.

14. Stambler BS. A new era of stroke prevention in atrial fibrillation: Comparing a new generation of oral anticoagulants with warfarin. Int Arch Med. 2013;6:46.

15. Altman R. New oral anticoagulants: Are coagulation units still required? Thromb J. 2014;12:3.

16. Tanaka-Esposito C, Chung MK. Selecting antithrombotic therapy for patients with atrial fibrillation. Cleve Clin J Med. 2015;82:49-63.

17. Ahrens I, Lip GY, Peter K. New oral anticoagulant drugs in cardiovascular disease. Thromb Haemost. 2010;104:49-60.

18. Connolly SJ, Ezekowitz MD, Yusuf S. Dabigatran versus warfarin in patients with atrial fibrillation. $N$ Engl J Med. 2009;361:1139-51.

19. Blacksmith C. Dabigatran: An alternative to warfarin. Cleveland Clinic. 2011;14:1-5.

20. Holy WE, Beer J. Update on the status of new oral anticoagulants for stroke prevention in patients with atrial fibrillation update on the status of new oral anticoagulants for stroke prevention in patients with atrial fibrillation. Cardiovascular Medicine. 2013;16:103-14.

21. Thachil J. The newer direct oral anticoagulants: A practical guide. Clin Med. 2014;14:165-75.

22. Mitchell SA, Simon TA, Raza S, et al. The efficacy and safety of oral anticoagulants in warfarin-suitable patients with nonvalvular atrial fibrillation: Systematic review and meta-analysis. Clin Appl Thromb Hemost. 2013;19:619-31.

23. Heidbuchel $H$, Verhamme $P$, Alings $M$, et al. European Heart Rhythm Association Practical Guide on the use of new oral anticoagulants in patients with non-valvular atrial fibrillation. Europace. 2013;15:625-51.

24. Chatterjee S, Sardar P, Giri JS, et al. Treatment discontinuations with new oral agents for long-term anticoagulation: Insights from a meta-analysis of 18 randomized trials including 101,801 patients. May Clin Proc. 2014;89:896-907.

25. Cove CL, Hylek EM. An updated review of target-specific oral anticoagulants used in stroke prevention in atrial fibrillation, venous thromboembolic disease, and acute coronary syndromes. J Am Heart Assoc. 2013;2:e000136.

26. You JJ, Singer DE, Howard PA, et al. Antithrombotic therapy for atrial fibrillation: Antithrombotic Therapy and Prevention 
of Thrombosis, 9th ed: American College of Chest Physicians Evidence-Based Clinical Practice Guidelines. Chest. 2012;141 2 Suppl, e531S-e575S.

27. Lip GY, Andreotti F, Fauchier L, et al. Bleeding risk assessment and management in atrial fibrillation patients: a position document from the European Heart Rhythm Association, endorsed by the European Society of Cardiology Working Group on Thrombosis. Europace. 2011;13:723-46.

28. Hankey GJ, Norrving B, Hacke W, et al. Management of acute stroke in patients taking novel oral anticoagulants. Int J Stroke. 2014;9:627-32.

29. Lazo-Langner A, Lang ES, Douketis J. Clinical review: Clinical management of new oral anticoagulants: A structured review with emphasis on the reversal of bleeding complications. Crit Care. 2013;17:230.

30. Siegal DM, Garcia DA, Crowther MA. How I treat targetspecific oral anticoagulant-associated bleeding. Blood. 2014;123:1152-8.

31. Jauch EC, Saber JL, Adams HP Jr, et al. Guidelines for the early management of patients with acute ischemic stroke: A guideline for healthcare professionals from the American Heart Association/American stroke Association. Stroke. 2013;44:870-947.

32. Komócsi A, Vorobcsuk A, Kehl D, et al. Use of new-generation oral anticoagulant agents in patients receiving antiplatelet therapy after an acute coronary syndrome: Systematic review and meta-analysis of randomized controlled trials. Arch Intern Med. 2012;172:1537-45.

33. Bassand JP. Novel oral anticoagulants in acute coronary syndrome: Re-evaluating the thrombin hypothesis. Eurolntervention. 2014;9:1333-41.

34. Costopoulos C, Niespialowska-Steuden M, Kukreja N, et al. Novel oral anticoagulants in acute coronary syndrome. Int J Cardiol. 2013;167:2449-55.

35. Liew A, Darvish-Kazem S, Douketis JD. Is there a role for novel oral anticoagulants in patients with an acute coronary syndrome? A review of the clinical trials. Pol Arch Med Wewn. 2013;123:617-22.

36. Dentali F, Botto GL, Gianni M, et al. Efficacy and safety of direct oral anticoagulants in patients undergoing cardioversion for atrial fibrillation: A systematic review and meta-analysis of the literature. Int J Cardiol. 2015;185:72-7.

37. Yadlapati A, Groh C, Passman R. Safety of short-term use of dabigatran or rivaroxaban for direct-current cardioversion in patients with atrial fibrillation and atrial flutter. Am J Cardiol. 2014;113:1362-3.

38. Nagarakanti R, Ezekowitz MD, Oldgren J, et al. Dabigatran versus warfarin in patients with atrial fibrillation: An analysis of patients undergoing cardioversion. Circulation. 2011;123:131-6.

39. Flaker G, Lopes RD, Al-Khatib SM, et al. Efficacy and safety of apixaban in patients after cardioversion for atrial fibrillation: insights from the ARISTOTLE Trial (Apixaban for Reduction in Stroke and Other Thromboembolic Events in Atrial Fibrillation). J Am Coll Cardiol. 2014;63:1082-7.

40. Piccini JP, Stevens SR, Lokhnygina Y, et al. Outcomes after cardioversion and atrial fibrillation ablation in patients treated with rivaroxaban and warfarin in the ROCKET AF trial. J Am Coll Cardiol. 2013;61:1998-2006.

41. Cappato R, Ezekowitz MD, Klein AL, et al. Rivaroxaban vs. vitamin $\mathrm{K}$ antagonists for cardioversion in atrial fibrillation. Eur Heart J. 2014;35:3346-55.

42. Yates SW. Novel oral anticoagulants for stroke prevention in atrial fibrillation: A focus on the older patient. Int J Gen Med. 2013;6:167-80.

43. Stöllberger C, Finsterer J. Concerns about the use of new oral anticoagulants for stroke prevention in elderly patients with atrial fibrillation. Drugs Aging. 2013;30:949-58.

44. Ng KH, Hart RG, Eikelboom JW. Anticoagulation in patients aged $\geq 75$ years with atrial fibrillation: Role of novel oral anticoagulants. Cardiol Ther. 2013;2:135-49.

45. Faraoni, Levy JH, Albaladejo P, et al. Updates in the perioperative and emergency management of non-vitamin $\mathrm{K}$ antagonist oral anticoagulants. Crit Care. 2015;19:203.

46. Lai A, Davidson N, Galloway SW, et al. Perioperative management of patients on new oral anticoagulants. $\mathrm{Br} \mathrm{J}$ Surg. 2014;101:742-9.

47. Kozek-Langenecker SA. Perioperative management issues of direct oral anticoagulants. Semin Hematol. 2014;51:112-20.

48. Deitelzweig $S$, Amin A, Jing $Y$, et al. Medical costs of oral anticoagulants vs warfarin for atrial fibrillation patients with different stroke risks. Cardiol Ther. 2013;2:165-70.

49. González-Juanatey JR, Álvarez-Sabin J, Lobos JM, et al. Análisis coste-efectividad de dabigatrán para la prevención de ictus y embolia sistémica en fibrilación auricular no valvular en España. Rev Esp Cardiol. 2012;65:901-10.

50. Wisløff T, Hagen G, Klemp M. Economic evaluation of warfarin, dabigatran, rivaroxaban, and apixaban for stroke prevention in atrial fibrillation. Pharmacoeconomics. 2014;32:601-12.

51. Mallouppas M, Vassiliou V. Anticoagulation for atrial fibrillation: Is this the end of warfarin? Not just yet. Journal of Angiology. 2013;2013:7.

52. Camm J, Colombo A, Corbucci G, et al. Left atrial appendage closure: A new technique for clinical practice. Heart Rhythm. 2014;11:514-21.

53. Masoudi F, Calkins H, Kavinsky C, et al. 2015 ACC/HRS/SCAl left atrial appendage occlusion device societal overview. Heart Rhythm. 2015;12:e122-36.

54. Reddy V, Doshi S, Sievert H, et al. Percutaneous left atrial appendage closure for stroke prophylaxis in patients with atrial fibrillation. 2.3-year follow-up of the PROTECT AF (Watchman Left Atrial Appendage System for Embolic Protection in Patients With Atrial Fibrillation) trial. Circulation. 2013;127:720-9. 